\title{
Rigidity and exotic models for the $K$-local stable homotopy category
}

\author{
CONSTANZE ROITZHEIM
}

\begin{abstract}
Can the model structure of a stable model category be recovered from the triangulated structure of its homotopy category? This paper introduces a new positive example for this, namely the $K$-local stable homotopy at the prime 2 . For odd primes, however, this is not true: we discuss a counterexample given by Jens Franke and show how such exotic models for the $K$-local stable homotopy category at odd primes can be detected.
\end{abstract}

55P42; 55P60

\section{Introduction}

When two model categories $\mathcal{C}$ and $\mathcal{D}$ are Quillen equivalent, then their homotopy categories $\operatorname{Ho}(\mathcal{C})$ and $\operatorname{Ho}(\mathcal{D})$ are equivalent. But on the other hand, if there is an equivalence between the homotopy categories of two model categories, can anything be said about the underlying model structures?

For the stable homotopy category $\operatorname{Ho}(\mathcal{S})$, that is, the homotopy category of spectra, there is the following result of Schwede [17]:

Rigidity Theorem (Schwede [17]) Let $\mathcal{C}$ be a stable model category, and

$$
\Phi: \operatorname{Ho}(\mathcal{S}) \longrightarrow \operatorname{Ho}(\mathcal{C})
$$

an equivalence of triangulated categories. Then the underlying model categories $\mathcal{S}$ and $\mathcal{C}$ are Quillen equivalent.

Usually, when passing from the model category level to the homotopy level, information can be lost, as "higher homotopy information" like mapping spaces or algebraic $K-$ theory is defined via the model structure of the underlying model category. However, the Rigidity Theorem says that for spectra, all such higher homotopy information is encoded in the triangulated structure of the stable homotopy category. 
Now the next question could be if there is a similar result for Bousfield localisations of the stable homotopy category with respect to certain homology theories. In this paper, we consider localisation with respect to 2-local complex $K$-theory $K_{(2)}$ with

$$
K_{(2) *}=\mathbb{Z}_{(2)}\left[v_{1}, v_{1}^{-1}\right], \quad\left|v_{1}\right|=2 .
$$

The $K_{(2)}$-local model structure is a model structure on the category of spectra where the weak equivalences are the $K_{(2) *}$-isomorphisms (see Definition 3.1). For the resulting $K_{(2)}$-local stable homotopy category we present the following positive answer to the rigidity question which is the main result of this paper:

$K_{(2)}$-local Rigidity Theorem Let $\mathcal{C}$ be a stable model category, let $L_{1} \mathcal{S}$ denote the $K$-local category of spectra at the prime 2 , and let

$$
\Phi: \operatorname{Ho}\left(L_{1} \mathcal{S}\right) \longrightarrow \operatorname{Ho}(\mathcal{C})
$$

be an equivalence of triangulated categories. Then $L_{1} \mathcal{S}$ and $\mathcal{C}$ are Quillen equivalent.

Remark The notation $L_{1}$ for $K_{(p)}$-localisation for a prime $p$ referes to the general context of chromatic localisation: the notation $L_{n}$ is often used to denote Bousfield localisation with respect to the Johnson-Wilson theories $E(n)$ with

$$
E(n)_{*}=\mathbb{Z}_{(p)}\left[v_{1}, v_{2}, \ldots, v_{n}, v_{n}^{-1}\right], \quad\left|v_{i}\right|=2 p^{i}-2,
$$

and in particular, $L_{K_{(p)}}=L_{E(1)}$.

The proof divides into two main parts. Firstly, we modify the Universal Property of Spectra introduced by Schwede and Shipley in $[18,5.1]$ to obtain a Quillen functor pair

$$
X \wedge-: L_{1} \mathcal{S} \rightleftarrows \mathcal{C}: \operatorname{Hom}(X,-)
$$

for $X=\Phi\left(L_{1} S^{0}\right)$ where $L_{1} S^{0}$ denotes the $K_{(2)}$-local sphere.

Secondly, we consider the left derived functor $X \wedge^{L}-$ composed with the inverse of $\Phi$

$$
\operatorname{Ho}\left(L_{1} \mathcal{S}\right) \stackrel{X \wedge^{L}-}{\longrightarrow} \operatorname{Ho}(\mathcal{C}) \stackrel{\Phi^{-1}}{\longrightarrow} \operatorname{Ho}\left(L_{1} \mathcal{S}\right) .
$$

It is an exact endofunctor of the homotopy category of $K_{(2)}$-local spectra, mapping the $K_{(2)}$-local sphere $L_{1} S^{0}$ to itself. The spectrum $L_{1} S^{0}$ is a so-called small weak generator of $L_{1} \mathcal{S}$. We show that any exact endofunctor fixing this small weak generator must be a self-equivalence, thus $X \wedge^{L}-$ is an equivalence of categories induced by a left Quillen functor. This means that $L_{1} \mathcal{S}$ and $\mathcal{C}$ are Quillen equivalent.

This paper is organised as follows: Section 2 gives a brief review of the relevant definitions. In Section 3, the Quillen pair $X \wedge-: L_{1} \mathcal{S} \rightleftarrows \mathcal{C}: \operatorname{Hom}(X,-)$ is constructed. 
We prove it to be a Quillen equivalence in Section 4. Next, Section 5 summarizes the necessary computations in the homotopy groups of the $K_{(2)}$-local sphere and the $K_{(2)}$-local mod-2 Moore spectrum.

As we explain in the last section, there cannot be an odd primary version of the $K_{(2)}-$ local Rigidity Theorem. For odd primes, Jens Franke constructed an equivalence of triangulated categories

$$
\mathcal{R}: \mathcal{D}^{2 p-2}(\mathcal{B}) \longrightarrow \operatorname{Ho}\left(L_{1} \mathcal{S}\right)
$$

between the homotopy category of $K$-local spectra at an odd prime $p$ and the derived category of so-called quasi-periodic cochain complexes over a certain abelian category $\mathcal{B}$ (see Franke [7, 3.1]). However, the underlying model categories $\mathcal{C}^{2 p-2}(\mathcal{B})$ and $L_{1} \mathcal{S}$ are not Quillen equivalent. This means that $\mathcal{C}^{2 p-2}(\mathcal{B})$ is a so-called "exotic model" for $L_{1} \mathcal{S}$. Last, we give a criterion whether a model for the $K_{(p)}$-local stable homotopy category is exotic or not.

As for a prime $p$ any algebraic model for $\operatorname{Ho}\left(L_{1} \mathcal{S}\right)$ is exotic, the $K_{(2)}$-local Rigidity Theorem implies that, in particular, the $K_{(2)}$-local stable homotopy category is not equivalent to the derived category of an abelian category.

\section{Acknowledgments}

First of all, I thank Stefan Schwede whose encouragement and interest in my work kept me highly motivated. Also, I thank Andy Baker for many helpful comments and discussions, and for inviting me to visit the University of Glasgow in June 2006. Further, I thank Hans-Werner Henn, Karlheinz Knapp, Mark Mahowald, Doug Ravenel, John Rognes and Sarah Whitehouse for suggestions and discussions. Finally, I am greatly indebted to this paper's referee for his careful reading and skillfully constructive criticism.

\section{Stable model categories - a review}

Model categories were introduced in the 1960s by Quillen to provide a set-theoretically clean device to describe homotopy [13]. A model category is a category equipped with classes of morphisms called weak equivalences, fibrations and cofibrations satisfying certain axioms (see, for example, Hovey $[11,1.1]$ ). These axioms enable us to define a notion of homotopy between morphisms.

Very roughly speaking, one then obtains the homotopy category $\operatorname{Ho}(\mathcal{C})$ of a model category $\mathcal{C}$ by formally inverting the weak equivalences, while the model category axioms ensure that the result is indeed a category. 
In order to compare model categories, one studies morphisms of model categories, so-called Quillen functors.

Definition 2.1 Let $\mathcal{C}$ and $\mathcal{D}$ be two model categories. An adjoint pair of functors $F: \mathcal{C} \rightleftarrows \mathcal{D}: G$ is called a Quillen functor pair if $F$ preserves cofibrations and trivial cofibrations (that is, cofibrations that are also weak equivalences), or equivalently, if $G$ preserves fibrations and trivial fibrations (that is, fibrations that are also weak equivalences).

Notation Throughout this paper, we use the following convention: for an adjoint functor pair $F: \mathcal{C} \rightleftarrows \mathcal{D}: G$, the top arrow denotes the left adjoint and the bottom arrow the right adjoint.

Cofibrations are marked $\longrightarrow$, fibrations $\longrightarrow$ and weak equivalences $\stackrel{\sim}{\longrightarrow}$.

If an adjoint pair of functors is a Quillen pair, it induces an adjoint pair of functors $L F: \operatorname{Ho}(\mathcal{C}) \rightleftarrows \operatorname{Ho}(\mathcal{D}): R G$ (see [11, Lemma 1.3.10]).

Definition 2.2 A Quillen functor pair is called a Quillen equivalence if in addition, for all cofibrant $X \in \mathcal{C}$ and fibrant $Y \in \mathcal{D}$, a morphism $f: F X \rightarrow Y$ is a weak equivalence if and only if its adjoint $\bar{f}: X \rightarrow G Y$ is.

One can conclude that a Quillen functor pair is a Quillen equivalence if and only if it induces an equivalence of homotopy categories [11, Prop. 1.3.13]. But not only do Quillen equivalent model categories have equivalent homotopy categories, they also have the "same homotopy theory" in the sense that the higher homotopy information mentioned in the introduction such as mapping spaces is preserved by Quillen equivalences.

For a pointed model category $\mathcal{C}$, one can define an adjoint pair of suspension and loop functors

$$
\Sigma: \operatorname{Ho}(\mathcal{C}) \rightleftarrows \operatorname{Ho}(\mathcal{C}): \Omega \text {. }
$$

Without loss of generality let $X \in \mathcal{C}$ be fibrant and cofibrant. We choose a factorisation

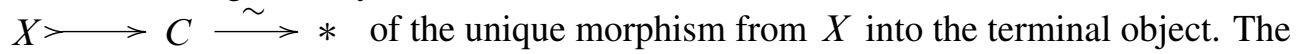
suspension $\Sigma X$ of $X$ is defined as the pushout of the diagram

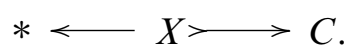

Dually, choosing a factorisation $* \stackrel{\sim}{\longrightarrow} A \longrightarrow X$, the loop functor $\Omega X$ of $X$ is defined as the pullback of the diagram

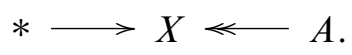


These constructions are not functorial or adjoint on $\mathcal{C}$, but they become functorial and adjoint in the homotopy category $\operatorname{Ho}(\mathcal{C})$.

Definition 2.3 A pointed, complete and cocomplete model category $\mathcal{C}$ is called stable if $\Sigma$ and $\Omega$ are inverse equivalences of homotopy categories.

Examples for stable model categories are provided by the category of spectra $\mathcal{S}$ (see the beginning of Section 3) or chain complexes $\mathcal{C}(\mathcal{A})$ for certain abelian categories $\mathcal{A}$.

The homotopy category $\operatorname{Ho}(\mathcal{C})$ of a stable model category $\mathcal{C}$ carries the structure of a triangulated category, where the exact triangles are given by the fiber and cofiber sequences $[11,7.1 .6]$.

In particular, the stable homotopy category $\operatorname{Ho}(\mathcal{S})$ and the derived category $\mathcal{D}(\mathcal{A})$ of an abelian category $\mathcal{A}$ are triangulated categories.

Furthermore, note the following: given a Quillen pair $F: \mathcal{C} \rightleftarrows \mathcal{D}: G$ with $\mathcal{C}$ and $\mathcal{D}$ being stable model categories, the left derived and right derived functors $L F$ and $R G$ are exact functors, that is, preserve exact triangles. This justifies the general rigidity question for stable model categories, namely, whether two stable model categories whose homotopy categories are equivalent as triangulated categories, are Quillen equivalent. However, the Rigidity Theorems for $\operatorname{Ho}(\mathcal{S})$ and $\operatorname{Ho}\left(L_{1} \mathcal{S}\right)$ do not claim that the given equivalence $\Phi$ is induced by a Quillen functor, they just claim that the given stable model categories are linked by some Quillen equivalence. The question about possible uniqueness of this Quillen functor is still unanswered.

\section{The Quillen functor pair}

\subsection{Universal property of spectra}

In this section, we construct a Quillen functor pair between the category of spectra equipped with the $K_{(2)}$-local model structure $L_{1} \mathcal{S}$ and our given stable model category $\mathcal{C}$.

Throughout this paper, $\mathcal{S}$ denotes the category of spectra with the stable BousfieldFriedlander model structure [4]. Here a spectrum $X$ is a sequence of pointed simplicial sets $\left(X_{0}, X_{1}, \ldots\right)$ together with structure maps $\sigma_{n}^{X}: \Sigma X_{n} \rightarrow X_{n+1}$. A morphism $f: X \rightarrow Y$ of spectra is a collection of morphisms of pointed simplicial sets $f_{n}: X_{n} \rightarrow$ $Y_{n}$ that are compatible with the structure maps, that is, $f_{n+1} \circ \sigma_{n}^{X}=\sigma_{n}^{Y} \circ \Sigma f_{n}$ for all $n \geq 0$. The $K_{(2)}$-local model structure on the category of spectra is a localisation of the Bousfield-Friedlander model structure: 
Definition 3.1 $\left(K_{(2)}\right.$-local model structure for spectra) A morphism of spectra $f: A \longrightarrow B$ is called a

- weak equivalence if $K_{(2) *} f: K_{(2) *}(A) \longrightarrow K_{(2) *}(B)$ is an isomorphism,

- cofibration, if the induced map

$$
\Sigma B_{n} \cup_{\Sigma A_{n}} A_{n+1} \longrightarrow B_{n+1}
$$

is a cofibration of simplicial sets for all $n \geq 1$ and $A_{0} \longrightarrow B_{0}$ is a cofibration of simplicial sets,

- fibration if $f$ has the right lifting property with respect to trivial cofibrations, that is, cofibrations that are also $K_{(2) *}$-isomorphisms.

Remark A spectrum $X$ is fibrant with respect to this model structure if and only if it is $K_{(2)}$-local in $\operatorname{Ho}(\mathcal{S})$ and an $\Omega$-spectrum.

With the above choices, the category of spectra becomes a stable model category, denoted by $L_{1} \mathcal{S}$. (For the definition of generalised Bousfield localisations see Hirschhorn [9, Definition 3.3.1]. For the existence of Bousfield localisations with respect to a generalised homology theory, see [9, Theorem 4.1.1]. The author believes that this theorem can be applied to this special case by using the set-theoretical methods of Bousfield [1, Sections 10-11]. However, the author does not know of any written reference for such a proof.)

Now, to construct our desired Quillen functor pair between $L_{1} \mathcal{S}$ and $\mathcal{C}$, we use the following.

Universal Property of Spectra (Schwede-Shipley [18]) Let $\mathcal{C}$ be a stable model category, and let $X \in \mathcal{C}$ be fibrant and cofibrant. Then there is a Quillen adjoint functor pair

$$
X \wedge-: \mathcal{S} \rightleftarrows \mathcal{C}: \operatorname{Hom}(X,-)
$$

where $X \wedge-$ sends the sphere spectrum $S^{0}$ to $X$.

Forgetting their model structures, $\mathcal{S}$ and $L_{1} \mathcal{S}$ are the same categories, so the above property gives us an adjoint pair of functors

$$
X \wedge-: L_{1} \mathcal{S} \rightleftarrows \mathcal{C}: \operatorname{Hom}(X,-)
$$

for any $X$. However, it is not obvious under which conditions this functor pair is a Quillen functor pair. 
Before we answer this, let us briefly summarize the construction of the functor

$$
\operatorname{Hom}(X,-): \mathcal{C} \longrightarrow \mathcal{S}
$$

For simplicity, let us assume $\mathcal{C}$ to be a pointed simplicial model category, that is, a category equipped with three functors

$$
\begin{array}{r}
-\otimes-: \mathcal{C} \times \operatorname{sSet} * \longrightarrow \mathcal{C} \\
(-)^{(-)}: \operatorname{sSet}^{o p} \times \mathcal{C} \longrightarrow \mathcal{C} \\
\operatorname{map}_{\mathcal{C}}(-,-): \mathcal{C}^{o p} \times \mathcal{C} \longrightarrow \operatorname{sSet} *
\end{array}
$$

satisfying certain adjunction properties and compatibilty with the model structure on $\mathcal{C}$. (For details, see Goerss and Jardine [8, Definition II.2.1].)

Notation In the pointed case, the first functor is usually denoted $-\wedge-$ instead of $-\otimes-$. However, we choose to write $-\otimes-$ to avoid confusion with the functor $X \wedge-$ as in the Universal Property of Spectra.

For $Y \in \mathcal{C}$, we define the $n^{\text {th }}$ level space of the spectrum $\operatorname{Hom}(X, Y)$ to be

$$
\operatorname{Hom}(X, Y)_{n}:=\operatorname{map}_{\mathcal{C}}\left(\omega^{n} X, Y\right) \in \operatorname{sSet}_{*}
$$

where $\omega^{n} X$ is a cofibrant replacement of an $n^{\text {th }}$ desuspension of $X$. We define $\omega^{n} X$ inductively by setting $\omega^{0} X=X$ and for $n \geq 1$ by choosing a factorisation

$$
\text { * } \omega^{n} X \underset{\varphi_{n}}{\stackrel{\sim}{\longrightarrow}} \Omega\left(\omega^{n-1} X\right) .
$$

By $\widetilde{\varphi_{n}}$ we denote the morphism $\Sigma \omega^{n} X \longrightarrow \omega^{n-1} X$ that is adjoint to $\varphi_{n}$. The structure map $\Sigma \operatorname{Hom}(X, Y)_{n-1} \longrightarrow \operatorname{Hom}(X, Y)_{n}$ of the spectrum $\operatorname{Hom}(X, Y)$ is now given by the adjoint of the map

$$
\operatorname{map}_{\mathcal{C}}\left(\omega^{n-1} X, Y\right) \stackrel{\operatorname{map}_{\mathcal{C}}\left(\widetilde{\varphi_{n}}, Y\right)}{\longrightarrow} \operatorname{map}_{\mathcal{C}}\left(\Sigma \omega^{n} X, Y\right) \simeq \Omega \operatorname{map}_{\mathcal{C}}\left(\omega^{n} X, Y\right) .
$$

As $\omega^{n} X$ is cofibrant in $\mathcal{C}$, the functor $\operatorname{map}_{\mathcal{C}}\left(\omega^{n} X,-\right): \mathcal{C} \longrightarrow$ sSet* preserves fibrations and trivial fibrations (see Hovey [11, Section 5]). One can conclude from this that the functor $\operatorname{Hom}(X,-): \mathcal{C} \longrightarrow \mathcal{S}$ preserves fibrations and trivial fibrations (as shown in Schwede and Shipley $[18,6.2])$. In particular, $\operatorname{Hom}(X, Y)$ is an $\Omega$-spectrum for fibrant $Y$, which is something we are going to make use of in the proof of the next proposition. 
Proposition 3.2 Let $\Phi: \operatorname{Ho}\left(L_{1} \mathcal{S}\right) \longrightarrow \operatorname{Ho}(\mathcal{C})$ be an equivalence of triangulated categories, and let $X$ be a cofibrant and fibrant object in $\mathcal{C}$ isomorphic to $\Phi\left(L_{1} S^{0}\right)$. Then

$$
X \wedge-: L_{1} \mathcal{S} \rightleftarrows \mathcal{C}: \operatorname{Hom}(X,-)
$$

is a Quillen functor pair with respect to the $K_{(2)}$-local model structure on the left side.

This proposition will be proven at the end of Section 3.2.

Notation Throughout the rest of this paper, $X$ will be a fixed fibrant and cofibrant replacement of $\Phi\left(L_{1} S^{0}\right)$. For a stable model category $\mathcal{D}$, and $A, B$ in $\mathcal{D},[A, B]_{*}^{\mathcal{D}}$ denotes the graded group of morphisms in the homotopy category of $\mathcal{D}$. All spectra are assumed to be 2-local, in particular $S^{0}=S_{(2)}^{0}$. By $M$ we denote the mod-2 Moore spectrum $M(\mathbb{Z} / 2)$.

\section{$3.2 v_{1}$-periodicity}

The key ingredient in the proof of the proposition is showing that the spectra $\operatorname{Hom}(X, Y)$ are $K_{(2)}$-local for all fibrant $Y \in \mathcal{C}$. A spectrum $A$ is $K_{(2)}$-local if and only if $v_{1}^{4}$ induces an isomorphism of its mod-2 homotopy groups $[M, A]_{*}^{\mathcal{S}}$ (see Bousfield [2, Section 4]). To be more precise:

For a prime $p$, let $K(1)$ denote the first Morava $K$-theory with

$$
K(1)_{*}=\mathbb{Z} / p\left[v_{1}, v_{1}^{-1}\right] .
$$

Any $p$-local finite spectrum $A$ with $H \mathbb{Q}_{*}(A)=0$ but $K(1)_{*}(A) \neq 0$ possesses a $v_{1}$-self map (see Hopkins and Smith [10, Section 3]), that is, a map

$$
v_{1}^{p^{i}}: \Sigma^{p^{i}(2 p-2)} A \longrightarrow A
$$

inducing an isomorphism in $K(1)$-homology. The notation is standard but slightly misleading since it seems to imply that $v_{1}^{p^{j}}$ is a power of an existing morphism $v_{1}$. However, this need not be the case:

In the case $p=2$, the mod-2 Moore spectrum $M$ has a $v_{1}$-self map

$$
v_{1}^{4}: \Sigma^{8} M \longrightarrow M
$$

that induces an isomorphism in $K(1)$-homology, or in this case equivalently, $K_{(2)}-$ homology. This is the smallest degree $v_{1}$-self map that can be realised on $M$. Also, this $v_{1}$-self map $v_{1}^{4}$ need not be unique, but our methods do not depend on this choice. 
Lemma 3.3 The map

$$
\left(v_{1}^{4}\right)^{*}:[M, \operatorname{Hom}(X, Y)]_{n}^{\mathcal{S}} \longrightarrow[M, \operatorname{Hom}(X, Y)]_{n+8}^{\mathcal{S}}
$$

is an isomorphism for all $n \in \mathbb{Z}$ and all $Y \in \mathcal{C}$. Thus, $\operatorname{Hom}(X, Y)$ is $K_{(2)}$-local for all $Y$.

Before we prove this lemma, we have to look at the image of certain elements in $\pi_{*} L_{1} S^{0}$ under the functor $X \wedge-$, namely the Hopf elements $\eta \in \pi_{1} L_{1} S^{0}, v \in$ $\pi_{3} L_{1} S^{0}$ and $\sigma \in \pi_{7} L_{1} S^{0}$, and further, the elements $y_{0} \in \pi_{0} L_{1} S^{0}, y_{1} \in \pi_{1} L_{1} S^{0}$ and $\mu \in \pi_{9} L_{1} S^{0}$.

(For details about the generators of the stable homotopy groups of the $K_{(2)}$-local sphere and their multiplicative relations see the table of generators of $\pi_{*} L_{1} S^{0}$ at the beginning of Section 5.1.)

Lemma 3.4 For $\eta, v, \sigma, y_{0}, y_{1}$, and $\mu$ in $\pi_{*} L_{1} S^{0}$ as before, we have

$$
\begin{array}{rlrl}
X \wedge \eta & =\Phi(\eta) \text { or } \Phi(\eta)+\Phi\left(y_{1}\right) & X \wedge \mu & =\Phi(\mu) \text { or } \Phi(\mu)+\Phi\left(\eta^{2} \sigma\right) \\
X \wedge v & =u \Phi(v) \text { for some odd } u \in \mathbb{Z} & X \wedge \sigma & =\bar{u} \Phi(\sigma) \text { for some odd } \bar{u} \in \mathbb{Z} \\
X \wedge y_{0} & =\Phi\left(y_{0}\right) & X \wedge y_{1} & =\Phi\left(y_{1}\right) .
\end{array}
$$

Notation Here, by $X \wedge-$ we actually mean the left derived functor $L(X \wedge-)$ of the Quillen functor $X \wedge-: \mathcal{S} \longrightarrow \mathcal{C}$ in the Universal Property of Spectra (see Schwede and Shipley [18]). However, we omit the $L$ in the notation for simplicity.

Since the proof of this lemma is rather long, we postpone it until a separate appendix. We continue with the proof of Lemma 3.3.

Proof We now prove Lemma 3.3, that is, that the mod-2 homotopy groups of $\operatorname{Hom}(X, Y)$ are $v_{1}^{4}$-periodic for all $Y \in \mathcal{C}$. By adjunction, it suffices to prove that

$$
\left(X \wedge v_{1}^{4}\right)^{*}:[X \wedge M, Y]_{n}^{\mathcal{C}} \longrightarrow\left[X \wedge \Sigma^{8} M, Y\right]_{n}^{\mathcal{C}}
$$

is an isomorphism for all integers $n$. Via the equivalence $\Phi$, the left and right side are isomorphic to $\left[M, \Phi^{-1}(Y)\right]_{n}^{L_{1} \mathcal{S}}$ and $\left[\Sigma^{8} M, \Phi^{-1}(Y)\right]_{n}^{L_{1} \mathcal{S}}$, respectively. Since $\left(v_{1}^{4}\right)^{*}$ is an isomorphism between these two groups, and therefore

$$
\Phi\left(v_{1}^{4}\right)^{*}:[X \wedge M, Y]_{n}^{\mathcal{C}} \longrightarrow\left[X \wedge \Sigma^{8} M, Y\right]_{n}^{\mathcal{C}}
$$

is an isomorphism, we will now investigate how $X \wedge v_{1}^{4}$ differs from $\Phi\left(v_{1}^{4}\right)$ by making use of the preceding lemma and the computations in Section 5. 
The element $X \wedge v_{1}^{4}$ lies in $\left[X \wedge \Sigma^{8} M, X \wedge M\right]_{0}^{\mathcal{C}}$, which, via $\Phi$ and Computation 5.7, is isomorphic to

$$
[M, M]_{8}^{L_{1} \mathcal{S}} \cong \mathbb{Z} / 4\left\{v_{1}^{4}\right\} \oplus \mathbb{Z} / 2\left\{\widetilde{\eta \sigma} \circ \text { pinch, } \operatorname{Id}_{L_{1} M} \wedge \eta \sigma\right\} .
$$

By Corollary 5.8, $2 v_{1}^{4}=$ incl $\circ \mu \circ$ pinch, so by Lemma 3.4, either

$$
\begin{aligned}
2\left(X \wedge v_{1}^{4}\right) & =(X \wedge \text { incl }) \circ(X \wedge \mu) \circ(X \wedge \text { pinch }) \\
& =\Phi(\text { incl }) \circ \Phi(\mu) \circ \Phi(\text { pinch }) \\
& =\Phi(\text { incl } \circ \mu \circ \text { pinch })=\Phi\left(2 v_{1}^{4}\right) \\
& =2 \Phi\left(v_{1}^{4}\right)
\end{aligned}
$$

or

$$
\begin{aligned}
2\left(X \wedge v_{1}^{4}\right) & =(X \wedge \text { incl }) \circ(X \wedge \mu) \circ(X \wedge \text { pinch }) \\
& =\Phi(\text { incl }) \circ\left(\Phi(\mu)+\Phi\left(\eta^{2} \sigma\right)\right) \circ \Phi(\text { pinch }) \\
& =\Phi(\text { incl } \circ \mu \circ \text { pinch })+0=\Phi\left(2 v_{1}^{4}\right) \\
& =2 \Phi\left(v_{1}^{4}\right)
\end{aligned}
$$

as either $X \wedge \mu=\Phi(\mu)$ or $X \wedge \mu=\Phi(\mu)+\Phi\left(\eta^{2} \sigma\right)$. (Note that incl $\circ \eta^{2} \sigma \circ$ pinch $=0$ by (3).)

This means that $X \wedge v_{1}^{4}$ can only differ from $\Phi\left(v_{1}^{4}\right)$ by an element of order at most two, that is,

$$
X \wedge v_{1}^{4}=\Phi\left(v_{1}^{4}\right)+\Phi(T), \quad \text { for some } \quad T \in[M, M]_{8}^{L_{1} \mathcal{S}}, \quad 2 T=0 .
$$

We will now show that all such $v_{1}^{4}+T$ are isomorphisms in $\operatorname{Ho}\left(L_{1} \mathcal{S}\right)$.

We will see that each $T \in[M, M]_{8}^{L_{1} \mathcal{S}}$ with $2 T=0$ induces the zero map in $K_{(2)}-$ homology. It is enough to check this for $2 v_{1}^{4}, \eta \sigma \circ$ pinch and $\operatorname{Id}_{L_{1} M} \wedge \eta \sigma$ as each $T$ in question is a sum of those.

The elements $\eta \in \pi_{1} L_{1} S^{0}$ and pinch $\in\left[M, S^{0}\right]_{-1}^{L_{1} \mathcal{S}}$ both induce the zero map in $K_{(2)}$-homology for degree reasons, as $K_{(2) *}\left(S^{0}\right)$ is concentrated in even degrees. Hence,

$K_{(2) *}\left(\operatorname{Id}_{L_{1} M} \wedge \eta \sigma\right), K_{(2) *}(\eta \sigma \circ$ pinch $) \quad$ and $\quad K_{(2) *}\left(2 v_{1}^{4}\right)=K_{(2) *}($ incl $\circ \mu \circ$ pinch $)$ are zero. (For the last equation, see Corollary 5.8.)

The element $v_{1}^{4}$ is of course a $K_{(2) *}$-isomorphism, and thus, each $v_{1}^{4}+T$ also is, as

$$
K_{(2) *}\left(v_{1}^{4}+T\right)=K_{(2) *}\left(v_{1}^{4}\right)+K_{(2) *}(T)=K_{(2) *}\left(v_{1}^{4}\right)+0=K_{(2) *}\left(v_{1}^{4}\right) .
$$


Consequently, every $v_{1}^{4}+T$ is an isomorphism in $\operatorname{Ho}\left(L_{1} \mathcal{S}\right)$. Hence, by adjunction,

$$
\left(v_{1}^{4}\right)^{*}:[M, \operatorname{Hom}(X, Y)]_{n}^{\mathcal{S}} \longrightarrow[M, \operatorname{Hom}(X, Y)]_{n+8}^{\mathcal{S}}
$$

is an isomorphism for all $n$ and $Y$, so $\operatorname{Hom}(X, Y)$ is a $K_{(2)}$-local spectrum for all $Y \in \mathcal{C}$.

Finally, we can prove Proposition 3.2, which says that for $X \cong \Phi\left(L_{1} S^{0}\right)$, the Universal Property of Spectra provides a Quillen functor pair between $L_{1} \mathcal{S}$ and $\mathcal{C}$.

Proof We show that the functor

$$
\operatorname{Hom}(X,-): \mathcal{C} \longrightarrow L_{1} \mathcal{S}
$$

is a right Quillen functor, that is, preserves fibrations and trivial fibrations.

Since the cofibrations in $\mathcal{S}$ are the same as in $L_{1} \mathcal{S}$, the left adjoint

$$
X \wedge-: L_{1} \mathcal{S} \longrightarrow \mathcal{C}
$$

preserves cofibrations because $X \wedge-: \mathcal{S} \longrightarrow \mathcal{C}$ is already a Quillen functor by the Universal Property of Spectra. Via adjunction it follows that

$$
\operatorname{Hom}(X,-): \mathcal{C} \longrightarrow L_{1} \mathcal{S}
$$

preserves trivial fibrations.

Now it remains to show that $\operatorname{Hom}(X,-)$ preserves fibrations. By Dugger [6, A.2] it suffices to show that $\operatorname{Hom}(X,-)$ preserves fibrations between fibrant objects. We do this in the following steps:

- for $Y \in \mathcal{C}$ fibrant, $\operatorname{Hom}(X, Y)$ is fibrant in $L_{1} \mathcal{S}$

- $\operatorname{Hom}(X,-)$ sends fibrations to level fibrations

- level fibrations between fibrant objects in $L_{1} \mathcal{S}$ are fibrations.

Let $Y \in \mathcal{C}$ be fibrant. Then, by Schwede and Shipley [18, 6.2], the spectrum $\operatorname{Hom}(X, Y)$ is an $\Omega$-spectrum, as also described at the beginning of Subsection 3.1. By Lemma 3.3, $\operatorname{Hom}(X, Y)$ is $K_{(2)}$-local. So since in $L_{1} \mathcal{S}$ the fibrant objects are exactly the $K_{(2)}$-local $\Omega$-spectra, $\operatorname{Hom}(X, Y)$ is fibrant for fibrant $Y$.

By construction, the functor $\operatorname{Hom}(X,-)$ sends fibrations to level fibrations, see [18, 6.2]. But level fibrations between fibrant objects are fibrations in $L_{1} \mathcal{S}$ : 
Let $A, B \in L_{1} \mathcal{S}$ be fibrant, and let $f: A \longrightarrow B$ be a level fibration. As both $A$ and $B$ are fibrant, $f$ is a fibration in $\mathcal{S}$. In $L_{1} \mathcal{S}$ we use the Factorisation Axiom to factor $f$ as the composite of a fibration and a trivial cofibration

$$
A \underset{i}{\stackrel{\sim}{\sim}} C \underset{p}{\longrightarrow} B .
$$

By assumption, $B$ is fibrant, and so must be $C$. So $i$ is a $K_{(2) *-\text { isomorphism between }}$ $K_{(2) *}$-local spectra and therefore a $\pi_{*}$-isomorphism. Also, $i$ is a cofibration in $\mathcal{S}$ since it is a cofibration in $L_{1} \mathcal{S}$, so $i$ is a trivial cofibration in $\mathcal{S}$.

Consequently, $i$ has the left lifting property in $\mathcal{S}$ with respect to the level fibration $f$

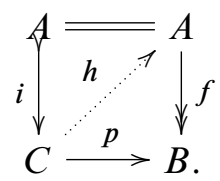

This gives us a commutative diagram in $L_{1} \mathcal{S}$

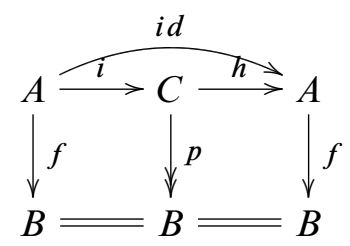

which says that $f$ is a retract of the $L_{1} \mathcal{S}$-fibration $p$ and therefore a fibration in $L_{1} \mathcal{S}$ by the Retract Axiom of model categories.

Putting these steps together, we have shown that $\operatorname{Hom}(X,-)$ is a right Quillen functor, which proves the proposition.

\section{The Quillen equivalence}

Again, let $\Phi: \operatorname{Ho}\left(L_{1} \mathcal{S}\right) \longrightarrow \operatorname{Ho}(\mathcal{C})$ be an equivalence of triangulated categories and let $X$ be a fibrant and cofibrant replacement of $\Phi\left(L_{1} S^{0}\right)$. In the last section we modified the Universal Property of Spectra (see Schwede and Shipley [18]) to construct a Quillen functor pair

$$
X \wedge-: L_{1} \mathcal{S} \rightleftarrows \mathcal{C}: \operatorname{Hom}(X,-) .
$$

This section will be devoted to showing that $(X \wedge-, \operatorname{Hom}(X,-))$ is a Quillen equivalence.

Notation The left derived functor of the Quillen functor $X \wedge-: L_{1} \mathcal{S} \longrightarrow \mathcal{C}$ will be denoted $X \wedge^{L}-$. 


\subsection{Homotopy type of $\operatorname{Hom}(X, X)$}

Our first goal is to show that $\operatorname{Hom}(X, X)$ is stably equivalent to the $K_{(2)}$-local sphere spectrum. Define

$$
\iota: S^{0} \longrightarrow \operatorname{Hom}(X, X)
$$

to be the morphism adjoint to the isomorphism $X \wedge S^{0} \cong X$. Since $\operatorname{Hom}(X, X)$ is $K_{(2)}$-local by Lemma 3.3, $\iota$ factors over the $K_{(2)}$-local sphere

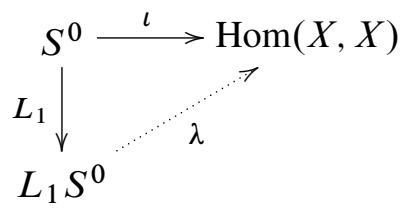

Proposition 4.1 The map $\lambda$ is a $\pi_{n}$-isomorphism for all integers $n$.

Proof We have the following diagram

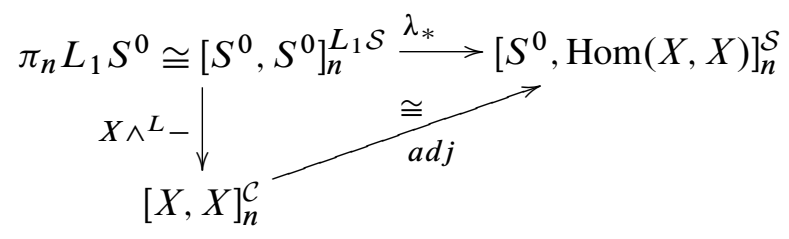

It is commutative because, by definition of $\lambda$, for $\alpha \in \pi_{*} L_{1} S^{0}$ the image of $X \wedge^{L} \alpha$ under the adjunction isomorphism is precisely $\lambda \circ \alpha$. Hence, $\lambda_{*}$ is an isomorphism if and only if

$$
X \wedge^{L}-:\left[S^{0}, S^{0}\right]_{n}^{L_{1} \mathcal{S}} \longrightarrow[X, X]_{n}^{\mathcal{C}}
$$

is an isomorphism. We show that

$$
\Psi:\left[S^{0}, S^{0}\right]_{n}^{L_{1} \mathcal{S}} \stackrel{X \wedge^{L}-}{\longrightarrow}[X, X]_{n}^{\mathcal{C}} \stackrel{\Phi^{-1}}{\longrightarrow}\left[S^{0}, S^{0}\right]_{n}^{L_{1} \mathcal{S}}
$$

is an isomorphism for all $n$.

The statement that $\Psi$ is an isomorphism in degree 0 through 9 follows directly from Lemma 3.4 (see also the table at the beginning of Section 5.1). Therefore, by adjunction, $\lambda_{*}$ is an isomorphism in degree 0 through 9 . It follows that $\lambda$ also induces an isomorphism between all rational homotopy groups of $L_{1} S^{0}$ and $\operatorname{Hom}(X, X)$ as these are concentrated in degree zero.

Using the exact triangle

$$
L_{1} S^{0} \stackrel{2}{\longrightarrow} L_{1} S^{0} \stackrel{\text { incl }}{\longrightarrow} L_{1} M \stackrel{\text { pinch }}{\longrightarrow} L_{1} S^{1}
$$


together with the 5-lemma, it follows that

$$
\Psi:\left[M, S^{0}\right]_{n}^{L_{1} \mathcal{S}} \longrightarrow\left[M, S^{0}\right]_{n}^{L_{1} \mathcal{S}}
$$

is an isomorphism for $n=0, \ldots, 8$. (We are still using our fixed isomorphism $X \wedge M \cong$ $\Phi\left(L_{1} M\right)$ which is omitted from our notation.)

Now $M$ has a $v_{1}$-self map $v_{1}^{4}$ that induces an isomorphism in $K_{(2)}$-homology, so

$$
v_{1}^{4}: \Sigma^{8} L_{1} M \longrightarrow L_{1} M
$$

is an isomorphism in $\operatorname{Ho}\left(L_{1} \mathcal{S}\right)$. Using the commutative diagram

$$
\begin{gathered}
{\left[M, S^{0}\right]_{n}^{L_{1} \mathcal{S}} \stackrel{\Psi}{\longrightarrow}\left[M, S^{0}\right]_{n}^{L_{1} \mathcal{S}}} \\
\left(v_{1}^{4}\right)^{*}\left|\cong \quad\left(\Psi\left(v_{1}^{4}\right)\right)^{*}\right| \cong \\
{\left[M, S^{0}\right]_{n+8}^{L_{1} \mathcal{S}} \stackrel{\Psi}{\longrightarrow}\left[M, S^{0}\right]_{n+8}^{L_{1} \mathcal{S}}}
\end{gathered}
$$

we obtain by induction that

$$
\Psi:\left[M, S^{0}\right]_{n}^{L_{1} \mathcal{S}} \longrightarrow\left[M, S^{0}\right]_{n}^{L_{1} \mathcal{S}}
$$

is an isomorphism for all integers $n$. (Note that the commutativity of the above diagram does not depend on the choice of the fixed isomorphisms $X \wedge M \cong \Phi\left(L_{1} M\right)$ and $X \wedge S^{0} \cong \Phi\left(L_{1} S^{0}\right)$.) Hence, by adjunction, $\lambda$ gives an isomorphism on the mod-2 homotopy groups of $L_{1} S^{0}$ and $\operatorname{Hom}(X, X)$.

But any map that induces isomorphisms between the rational homotopy groups and the mod-2 homotopy groups of 2-local spectra must be a weak equivalence. Thus,

$$
\lambda_{*}: \pi_{n} L_{1} S^{0} \longrightarrow \pi_{n} \operatorname{Hom}(X, X)
$$

is an isomorphism for all $n$, so $L_{1} S^{0}$ and $\operatorname{Hom}(X, X)$ are stably equivalent via $\lambda$.

With this, we can now prove the $K_{(2)}$-local Rigidity Theorem:

\subsection{Proof of the Main Theorem}

Theorem 4.2 Let $\Phi: \operatorname{Ho}\left(L_{1} \mathcal{S}\right) \longrightarrow \operatorname{Ho}(\mathcal{C})$ be an equivalence of triangulated categories, $X$ a fibrant and cofibrant replacement of $\Phi\left(L_{1} S^{0}\right)$, and

$$
X \wedge-: L_{1} \mathcal{S} \rightleftarrows \mathcal{C}: \operatorname{Hom}(X,-)
$$

the Quillen functor pair from Proposition 3.2. Then $(X \wedge-, \operatorname{Hom}(X,-))$ is a Quillen equivalence. 
Proof By Hovey $[11,1.3 .16]$, it suffices to show the following:

(1) $\operatorname{RHom}(X,-): \operatorname{Ho}(\mathcal{C}) \longrightarrow \operatorname{Ho}\left(L_{1} \mathcal{S}\right)$ reflects isomorphisms

(2) The map $A \longrightarrow \operatorname{RHom}\left(X, X \wedge^{L} A\right)$ is an isomorphism for all $A \in \operatorname{Ho}\left(L_{1} \mathcal{S}\right)$.

(Here $\operatorname{RHom}(X,-)$ denotes the right derived functor of the Quillen functor $\operatorname{Hom}(X,-): \mathcal{C} \longrightarrow L_{1} \mathcal{S}$.)

To prove these two points, we make use of the fact that $\operatorname{Ho}\left(L_{1} \mathcal{S}\right)$ is a compactly generated triangulated category:

The $K_{(2)}$-local sphere is a small weak generator in $\operatorname{Ho}\left(L_{1} \mathcal{S}\right)$, that is, $\left[S^{0},-\right]^{L_{1} \mathcal{S}}$ commutes with coproducts and detects isomorphisms. So by Keller [12, 4.2], any triangulated subcategory of $\operatorname{Ho}\left(L_{1} \mathcal{S}\right)$ that is closed under coproducts and containing the sphere must already be $\operatorname{Ho}\left(L_{1} \mathcal{S}\right)$ itself. Since $\Phi$ is an equivalence of triangulated categories, $\Phi\left(L_{1} S^{0}\right)=X$ is a small weak generator for $\operatorname{Ho}(\mathcal{C})$, that is, any triangulated subcategory of $\operatorname{Ho}(\mathcal{C})$ that is closed under coproducts and containing $X$ is again $\operatorname{Ho}(\mathcal{C})$ itself.

Let us first show that $\operatorname{RHom}(X,-)$ reflects isomorphisms. For a morphism $f: Y \longrightarrow$ $Z$ in $\mathcal{C}$, let $\operatorname{RHom}(X, f): \operatorname{RHom}(X, Y) \longrightarrow \operatorname{RHom}(X, Z)$ be an isomorphism in $\operatorname{Ho}\left(L_{1} \mathcal{S}\right)$, so

$$
\left[S^{0}, \operatorname{RHom}(X, Y)\right]_{*}^{L_{1} \mathcal{S}} \stackrel{\operatorname{RHom}(X, f)}{\longrightarrow}\left[S^{0}, \operatorname{RHom}(X, Z)\right]_{*}^{L_{1} \mathcal{S}}
$$

is an isomorphism. By adjunction,

$$
[X, Y]_{*}^{\mathcal{C}} \stackrel{f_{*}}{\longrightarrow}[X, Z]_{*}^{\mathcal{C}}
$$

is an isomorphism.

But as $X$ is a generator in $\operatorname{Ho}(\mathcal{C})$ (see above), it detects isomorphisms, so

$$
f: Y \longrightarrow Z
$$

is an isomorphism in $\operatorname{Ho}(\mathcal{C})$ which proves the first point.

To prove the second point, we define $\mathcal{T}$ to be the full subcategory of $\operatorname{Ho}\left(L_{1} \mathcal{S}\right)$ containing those $A \in \operatorname{Ho}\left(L_{1} \mathcal{S}\right)$ such that

$$
A \longrightarrow \operatorname{RHom}\left(X, X \wedge^{L} A\right)
$$

is an isomorphism. We want to prove that $\mathcal{T}=\operatorname{Ho}\left(L_{1} \mathcal{S}\right)$. 
Since $\operatorname{RHom}(X,-)$ and $X \wedge^{L}-$ are exact functors, $\mathcal{T}$ is triangulated. By Proposition 4.1, $L_{1} S^{0} \in \mathcal{T}$. Now let $A_{i}, i \in I$, be a family of objects in $\mathcal{T}$. We want to prove that $\bigsqcup_{i \in I} A_{i} \in \mathcal{T}$. By adjunction,

$$
\left[S^{0}, \operatorname{RHom}\left(X, X \wedge^{L}\left(\coprod_{i} A_{i}\right)\right)\right]_{*}^{L_{1} \mathcal{S}} \cong\left[X, X \wedge^{L}\left(\coprod_{i} A_{i}\right)\right]_{*}^{\mathcal{C}} .
$$

As a left adjoint, $X \wedge^{L}-$ commutes with coproducts, so

$$
\left[X, X \wedge^{L}\left(\coprod_{i} A_{i}\right)\right]_{*}^{\mathcal{C}} \cong\left[X, \coprod_{i}\left(X \wedge^{L} A_{i}\right)\right]_{*}^{\mathcal{C}} \text {. }
$$

Since $X \cong \Phi\left(L_{1} S^{0}\right)$ is small, we have

$$
\left[X, \coprod_{i}\left(X \wedge^{L} A_{i}\right)\right]_{*}^{\mathcal{C}} \cong \bigoplus_{i}\left[X, X \wedge^{L} A_{i}\right]_{*}^{\mathcal{C}} \cong \bigoplus_{i}\left[S^{0}, \operatorname{RHom}\left(X, X \wedge^{L} A_{i}\right)\right]_{*}^{L_{1} \mathcal{S}}
$$

As $A_{i} \in \mathcal{T}$ for all $i$,

$$
\left[S^{0}, A_{i}\right]_{*}^{L_{1} \mathcal{S}} \cong\left[S^{0}, \operatorname{RHom}\left(X, X \wedge^{L} A_{i}\right)\right]_{*}^{L_{1} \mathcal{S}},
$$

induced by

$$
A_{i} \stackrel{\cong}{\rightarrow} \operatorname{RHom}\left(X, X \wedge^{L} A_{i}\right) .
$$

So by naturality of the preceding isomorphisms,

$$
\left[S^{0}, \coprod_{i} A_{i}\right]_{*}^{L_{1} \mathcal{S}} \cong\left[S^{0}, \operatorname{RHom}\left(X, X \wedge^{L}\left(\coprod_{i} A_{i}\right)\right)\right]_{*}^{L_{1} \mathcal{S}}
$$

is an isomorphism induced by the map

$$
\coprod_{i} A_{i} \longrightarrow \operatorname{RHom}\left(X, X \wedge^{L}\left(\coprod_{i} A_{i}\right)\right) \text {. }
$$

Since the $K_{(2)}$-local sphere detects isomorphisms, this map is an isomorphism in $\operatorname{Ho}\left(L_{1} \mathcal{S}\right)$.

So we have seen that $\mathcal{T}$ is triangulated, contains $L_{1} S^{0}$ and is closed under coproducts.

As $\operatorname{Ho}\left(L_{1} \mathcal{S}\right)$ is compactly generated with generator $L_{1} S^{0}$, every triangulated subcategory of $\operatorname{Ho}\left(L_{1} \mathcal{S}\right)$ containing $L_{1} S^{0}$ that is closed under coproducts must already be $\operatorname{Ho}\left(L_{1} \mathcal{S}\right)$ itself by the criterion of Keller mentioned earlier, so $\mathcal{T}=\operatorname{Ho}\left(L_{1} \mathcal{S}\right)$. This means that

$$
A \longrightarrow \operatorname{RHom}\left(X, X \wedge^{L} A\right)
$$

is an isomorphism for all $A \in \operatorname{Ho}\left(L_{1} \mathcal{S}\right)$. 
We can now conclude that $(X \wedge-, \operatorname{Hom}(X,-))$ is a Quillen equivalence for $X \cong$ $\Phi\left(L_{1} S^{0}\right)$. So, given an equivalence of triangulated categories

$$
\Phi: \operatorname{Ho}\left(L_{1} \mathcal{S}\right) \longrightarrow \operatorname{Ho}(\mathcal{C})
$$

we have proven that $\mathcal{C}$ and $L_{1} \mathcal{S}$ are Quillen equivalent, which proves the $K$-local Rigidity Theorem at the prime 2 .

\section{Computations}

For our main proofs we need information about $[M, M]_{*}^{L_{1} \mathcal{S}}$ and $\left[S^{0}, M\right]_{*}^{L_{1} \mathcal{S}}$ in certain degrees. The necessary computations will be summarized in this section.

\subsection{Generators and relations of $\pi_{*} L_{1} S^{0}$}

First, let us look at the homotopy groups of the $K_{(2)}$-local sphere (see, for example, Bousfield [2, Proposition 4.5] or Ravenel [14, 8.15]). The ring homomorphism

$$
L_{1}: \pi_{*} S^{0} \longrightarrow \pi_{*} L_{1} S^{0}
$$

induced by $K_{(2)}$-localisation is surjective in degrees $\geq 2$, and it has a cokernel isomorphic to $\mathbb{Z} / 2$ in degrees 0 and 1 . There is a unique order 2 element of $\pi_{0} L_{1} S^{0}$ called $y_{0}$, and $y_{1}=\eta y_{0}$ is a generator of the second $\mathbb{Z} / 2$ summand in $\pi_{1} L_{1} S^{0}$. The other elements of $\pi_{*} L_{1} S^{0}$ are given the names of their (not necessarily unique) preimage in $\pi_{*} S^{0}$. So in low degrees we have the elements shown in Table 1.

\begin{tabular}{|c|c||c|c|}
\hline$k$ & $\pi_{k} L_{1} S^{0}$ & $k$ & $\pi_{k} L_{1} S^{0}$ \\
\hline 0 & $\mathbb{Z}_{(2)}\{l\} \oplus \mathbb{Z} / 2\left\{y_{0}\right\}$ & 5 & 0 \\
1 & $\mathbb{Z} / 2\left\{\eta, y_{1}\right\}$ & 6 & 0 \\
2 & $\mathbb{Z} / 2\left\{\eta^{2}\right\}$ & 7 & $\mathbb{Z} / 16\{\sigma\}$ \\
3 & $\mathbb{Z} / 8\{v\}$ & 8 & $\mathbb{Z} / 2\{\eta \sigma\}$ \\
4 & 0 & 9 & $\mathbb{Z} / 2\left\{\eta^{2} \sigma, \mu\right\}$ \\
\hline
\end{tabular}

Table 1

Moreover, we have

$$
4 v=\eta^{3}, \eta y_{1}=0, y_{0}^{2}=0, y_{1}^{2}=0, \sigma y_{1}=0 \quad \text { and } \quad \mu y_{0}=\eta^{2} \sigma
$$


by Ravenel $[14,8.15$.(d)]. Furthermore, we make use of the following Toda bracket relations:

$$
\begin{aligned}
& 8 \sigma=\langle\nu, 8, v\rangle \\
& \mu \in\langle 2,8 \sigma, \eta\rangle \quad \text { (indeterminacy: } \eta^{2} \sigma \text { ) }
\end{aligned}
$$

The element $\mu$ is the unique element of the second Toda bracket with Adams filtration five. For a reference for the relations, see Toda [19, Lemma 5.13, Lemma 10.9, tables in Chapter XIV].

Notation Throughout this paper, we read Toda brackets from right to left, that is, in the same direction as the composition of morphisms.

\subsection{Homotopy groups and endomorphisms of $L_{1} M$}

We will now compute some homotopy groups of the $K_{(2)}$-local mod-2 Moore spectrum. The long exact homotopy sequence of the exact triangle

$$
L_{1} S^{0} \stackrel{2}{\longrightarrow} L_{1} S^{0} \stackrel{\text { incl }}{\longrightarrow} L_{1} M \stackrel{\text { pinch }}{\longrightarrow} L_{1} S^{1}
$$

splits into short exact sequences of the form

$$
0 \longrightarrow \pi_{m+1} L_{1} S^{0} /(2) \stackrel{\text { incl }_{*}}{\longrightarrow} \pi_{m+1} L_{1} M \stackrel{\text { pinch }_{*}}{\longrightarrow}\left\{\pi_{m} L_{1} S^{0}\right\}_{2} \longrightarrow 0 .
$$

Here, $\left\{\pi_{m} L_{1} S^{0}\right\}_{2}$ denotes the 2-torsion of the group $\pi_{m} L_{1} S^{0}$, that is, all $x \in$ $\pi_{m} L_{1} S^{0}$ with $2 x=0$.

Let $x \in\left\{\pi_{m} L_{1} S^{0}\right\}_{2}$, and $\tilde{x} \in \pi_{m+1} L_{1} M$ a lift of $x$, that is, an element with pinch $\circ \tilde{x}=x$. We have $\operatorname{pinch}_{*}(2 \tilde{x})=0$, so $2 \tilde{x}$ has a unique preimage under the map incl ${ }_{*}$. This preimage is $\eta x \in \pi_{m+1} L_{1} S^{0} /(2)$, as

$$
\operatorname{incl}_{*}(\eta x)=(\text { incl } \circ \eta \circ \text { pinch }) \circ \tilde{x}=2 \tilde{x},
$$

remembering incl $\circ \eta \circ$ pinch $=2 \operatorname{Id}_{L_{1} M}$

Notation A preimage of an element $x$ under the pinch map will be denoted by $\tilde{x}$. This $\tilde{x}$ need not be unique, but the following computations do not depend on the choice of such an $\tilde{x}$ unless stated.

For some particular examples this gives us

Computation $5.1 \quad \pi_{0} L_{1} M \cong \mathbb{Z} / 2\left\{\right.$ incl, incl $\left.\circ y_{0}\right\}$ 
Computation $5.2 \pi_{1} L_{1} M \cong \mathbb{Z} / 4\left\{\tilde{y_{0}}\right\} \oplus \mathbb{Z} / 2\{$ incl $\circ \eta\}$

Computation $5.3 \pi_{8} L_{1} M \cong \mathbb{Z} / 2\{$ incl $\circ \eta \sigma, \widetilde{8 \sigma}\}$

Computation $5.4 \pi_{9} L_{1} M \cong \mathbb{Z} / 4\{\widetilde{\eta \sigma}\} \oplus \mathbb{Z} / 2\{$ incl $\circ \mu\}$

Also, note that

$$
\text { incl } \circ y_{1} \circ \text { pinch }=\text { incl } \circ \eta y_{0} \text { pinch }=2 \tilde{y_{0}} \text { pinch }=\tilde{y_{0}}(2 \text { pinch })=0,
$$

and

$$
\text { incl } \circ \eta^{2} \sigma \circ \text { pinch }=2 \widetilde{\eta \sigma} \text { pinch }=\widetilde{\eta \sigma}(2 \text { pinch })=0 .
$$

To specify the element $\widetilde{8 \sigma}$ in Computation 5.3 and for further applications we need the following:

Lemma 5.5 $8 \sigma=$ pinch $\circ v_{1}^{4} \circ$ incl in $\operatorname{Ho}\left(L_{1} \mathcal{S}\right)$.

Proof The element pinch $\circ v_{1}^{4} \circ$ incl lies in $\pi_{7} L_{1} S^{0}$. Since 2 pinch $=0$, it has order at most two, so the element in question is either $8 \sigma$ or 0 .

Assume that pinch $\circ v_{1}^{4} \circ$ incl $=0$, then $v_{1}^{4} \circ$ incl factors over the fiber of the pinch map, which, after $K_{(2)}$-localisation, gives us the commutative diagram

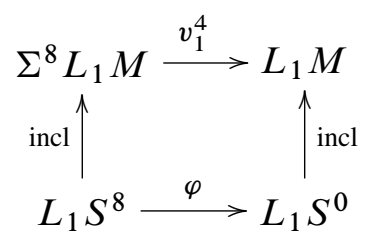

The element $\varphi$ lies in $\pi_{8} L_{1} S^{0} \cong \mathbb{Z} / 2\{\eta \sigma\}$, so $\varphi=\eta a$ for either $a=0$ or $a=\sigma$. We now apply the $m^{t h} K(1)$-homology to the above diagram, using this factorisation of $\varphi$ :

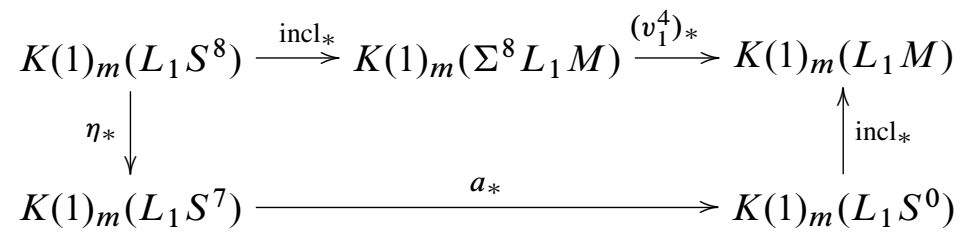

For even $m, \operatorname{incl}_{*}=K(1)_{m}(\mathrm{incl})$ is an isomorphism, and the map $v_{1}^{4}$ is a $K(1)_{*^{-}}$ isomorphism, so the upper row is an isomorphism for even $m$. However, $\eta_{*}$ lowers the degree by one, so it must be zero since the $K(1)$-homology of the sphere is concentrated in even degrees. Thus, we have arrived at a contradiction. 
So since there is no $\varphi \in \pi_{8} L_{1} S^{0}$ with incl $\circ \varphi=v_{1}^{4} \circ$ incl, the composition pinch $\circ v_{1}^{4} \circ$ incl $\in \pi_{7} S^{0}$ is nonzero, has order two and therefore must be $8 \sigma$.

Corollary 5.6 $\pi_{8} L_{1} M \cong \mathbb{Z} / 2\left\{\right.$ incl $\circ \eta \sigma, v_{1}^{4} \circ$ incl $\}$

Computation $5.7[M, M]_{8}^{L_{1} \mathcal{S}} \cong \mathbb{Z} / 4\left\{v_{1}^{4}\right\} \oplus \mathbb{Z} / 2\left\{\widetilde{\eta \sigma} \circ\right.$ pinch, $\left.\operatorname{Id}_{L_{1} M} \wedge \eta \sigma\right\}$

Proof We consider the short exact sequence

$$
0 \longrightarrow \pi_{9} L_{1} M /(2) \stackrel{\text { pinch* }^{*}}{\longrightarrow}[M, M]_{8}^{L_{1} \mathcal{S}} \stackrel{\text { incl* }^{*}}{\longrightarrow}\left\{\pi_{8} L_{1} M\right\}_{2} \longrightarrow 0 .
$$

Let $x \in\left\{\pi_{8} L_{1} M\right\}_{2}, \bar{x} \in[M, M]_{8}^{L_{1} \mathcal{S}}$ with incl* $(\bar{x})=\bar{x}$ oincl $=x$. Since $2 \operatorname{Id}_{L_{1} M} x=$ 0 , the element $2 \bar{x}$ has a unique preimage $q \in \pi_{9} L_{1} M /(2)$. This $q$ lies in the Toda bracket $\left\langle 2 \operatorname{Id}_{L_{1} M}, x, 2\right\rangle$ :

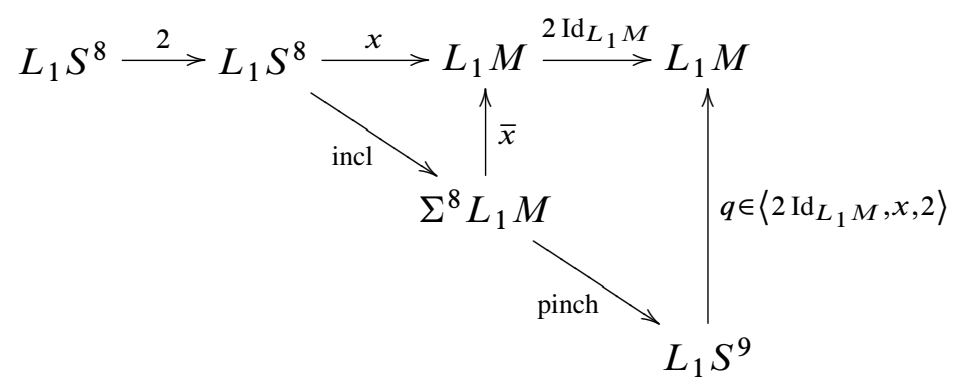

So to determine whether $2 \bar{x}=0$ for any $x \in\left\{\pi_{8} L_{1} M\right\}_{2}$, we have to compute the brackets $\left\langle 2 \operatorname{Id}_{L_{1} M}, v_{1}^{4} \circ\right.$ incl, 2$\rangle$ and $\left\langle 2 \operatorname{Id}_{L_{1} M}\right.$, incl $\left.\varnothing \eta \sigma, 2\right\rangle$.

Since $2 \operatorname{Id}_{L_{1} M}=$ incl $\circ \eta \circ$ pinch, the first bracket can be written as

$$
\begin{aligned}
\left\langle 2 \operatorname{Id}_{L_{1} M}, v_{1}^{4} \circ \text { incl, } 2\right\rangle & =\left\langle\text { incl } \circ \eta \circ \text { pinch, } v_{1}^{4} \circ \text { incl, } 2\right\rangle \\
& =\left\langle\text { incl } \circ \eta, \text { pinch } \circ v_{1}^{4} \circ \text { incl, } 2\right\rangle \\
& =\langle\text { incl } \circ \eta, 8 \sigma, 2\rangle \\
& =\text { incl } \circ\langle\eta, 8 \sigma, 2\rangle
\end{aligned}
$$

where the second and fourth equality are due to the Juggling Theorem (see Ravenel [15, A1.4.6]) and the third equality is due to Lemma 5.5. This means that $2 v_{1}^{4}$ is hit in the short exact sequence by incl $\circ \mu$ or incl $\circ\left(\mu+\eta^{2} \sigma\right)$, as $\mu \in\langle\eta, 8 \sigma, 2\rangle$ with indeterminacy $\eta^{2} \sigma$. Since

$$
\text { incl } \circ \eta^{2} \sigma \circ \text { pinch }=2 \widetilde{\eta \sigma} \text { pinch }=0 \quad \text { by }(3),
$$


we have in either case

$$
2 v_{1}^{4}=\text { incl } \circ \mu \circ \text { pinch } \neq 0 .
$$

The second bracket gives us

$$
\begin{aligned}
\left\langle 2 \operatorname{Id}_{L_{1} M}, \text { incl } \circ \eta \sigma, 2\right\rangle & =\langle\text { incl } \circ \eta, \text { pinch } \circ \text { incl } \circ \eta \sigma, 2\rangle \\
& =\langle\text { incl } \circ \eta, 0,2\rangle=0 .
\end{aligned}
$$

The indeterminacy here is $2 \pi_{9} L_{1} M$, that is, zero in $\pi_{9} L_{1} M /(2)$. Applying these computations to the short exact sequence (4) gives us now the desired result: we now know that $[M, M]_{8}^{L_{1} \mathcal{S}} \cong \mathbb{Z} / 4 \oplus \mathbb{Z} / 2 \oplus \mathbb{Z} / 2$ with $v_{1}^{4}$ generating the $\mathbb{Z} / 4$ summand, and $\tilde{\eta \sigma} \circ$ pinch generating one of the $\mathbb{Z} / 2$ summands. Looking at the sequence (4) again we see that any element $P \in[M, M]_{8}^{L_{1} \mathcal{S}}$ with $P \circ$ incl $=$ incl $\circ \eta \sigma$ can be taken to be a generator of the other $\mathbb{Z} / 2$ summand, so we choose $P=\operatorname{Id}_{L_{1}} M \wedge \eta \sigma$ (where $\wedge$ denotes the smash product in the homotopy category of $\mathcal{S}$ ).

Corollary $5.82 v_{1}^{4}=$ incl $\circ \mu \circ$ pinch $\neq 0$.

\section{The case against odd primes}

As mentioned in the introduction, in the case of $p>2$, rigidity for $K_{(p)}$-local spectra cannot hold because of an example constructed by Franke in [7]. In this section, we give a brief review of this exotic model and explain where the proof of the $K_{(2)}$-local Rigidity Theorem must fail when replacing 2 by an odd prime $p$.

\subsection{Franke's exotic models}

Throughout the rest of this section, let $p$ denote an odd prime. Franke proves that the homotopy category of $K_{(p)}$-local spectra is triangulated equivalent to the derived category of $(2 p-2)$-twisted cochain complexes over a certain abelian category $\mathcal{B}$.

Theorem 6.1 (Franke [7]) There is an equivalence of categories

$$
\mathcal{R}: \mathcal{D}^{2 p-2}(\mathcal{B}) \longrightarrow \operatorname{Ho}\left(L_{1} \mathcal{S}\right)
$$

where $\mathcal{D}^{2 p-2}(\mathcal{B})$ denotes the derived category of twisted cochain complexes over an abelian category $\mathcal{B}$, and $\operatorname{Ho}\left(L_{1} \mathcal{S}\right)$ the homotopy category of $K_{(p)}$-local spectra for an odd prime $p$.

We are now going to explain the ingredients of this theorem. We begin with the abelian categories $\mathcal{A}$ and $\mathcal{B}$. The category $\mathcal{B}$ consists of $\mathbb{Z}_{(p)}$-modules together with the action 
of Adams operations $\psi^{k}, k \in \mathbb{Z}_{(p)}^{*}$, satisfying some further conditions. (Details can be found in Bousfield [3] or Franke [7, 3.1], see also Clarke, Crossley and Whitehouse [5].)

To build the category $\mathcal{A}$ out of the above category, we additionally need the following: Let $T: \mathcal{B} \longrightarrow \mathcal{B}$ denote the following self-equivalence:

For all $M \in \mathcal{B}, \quad T(M)=M \quad$ as a $\mathbb{Z}_{(p)}$-module, but on $T(M)$, the Adams operation $\psi^{k}$ now equals $k^{p-1} \psi^{k}: M \longrightarrow M$ for all $k \in \mathbb{Z}$.

An object $\mathcal{M} \in \mathcal{A}$ is defined as a collection of modules $\mathcal{M}=\left(M_{n}\right)_{n \in \mathbb{Z}}$, where $M_{n} \in \mathcal{B}$, together with isomorphisms

$$
T\left(M_{n}\right) \stackrel{\cong}{\longrightarrow} M_{n+2 p-2} \text { for all } n \in \mathbb{Z} .
$$

The resulting category $\mathcal{A}$ is equivalent to the category of $E(1)_{*} E(1)$-comodules, with $E(1)$ denoting the Adams summand of $p$-local $K$-theory with coefficient groups

$$
E(1)_{*} \cong \mathbb{Z}_{(p)}\left[v_{1}, v_{1}^{-1}\right],\left|v_{1}\right|=2 p-2 .
$$

Note the following: For $X$ a spectrum, the $E(1)_{*} E(1)$-comodule $E(1)_{*}(X)$ is an object of $\mathcal{A}$ in the above sense by taking $M_{n}:=E(1)_{n}(X)$, and the operations $\psi^{k}$ being the usual Adams operations.

From now on $\mathcal{B}$ will be viewed as the subcategory of $\mathcal{A}$ consisting of those objects $\left(M_{n}\right)_{n \in \mathbb{Z}}$ such that

$$
M_{n}=\left\{\begin{aligned}
M & \text { if } n \equiv 0 \bmod 2 p-2 \\
0 & \text { otherwise }
\end{aligned}\right.
$$

for a $\mathbb{Z}_{(p)}$-module $M$ with Adams operations as before. This describes a so-called split of period $2 p-2$ of $\mathcal{A}: \mathcal{B} \subset \mathcal{A}$ is a Serre class such that

$$
\begin{gathered}
\bigoplus_{0 \leq i<2 p-2} \mathcal{B} \longrightarrow \mathcal{A} \\
\left(B_{i}\right)_{0 \leq i<2 p-2} \longmapsto \bigoplus_{0 \leq i<2 p-2} B_{i}[i]
\end{gathered}
$$

is an equivalence of categories, where $[i]$ denotes the $i$-fold internal shift in the grading, that is, $M[i]_{n}=M_{i-n}$.

Now we describe the source of Franke's equivalence.

Definition 6.2 The category $\mathcal{C}^{2 p-2}(\mathcal{B})$ of twisted cochain complexes with values in $\mathcal{B}$ is defined as follows: 
An object is a cochain complex $C^{*}$ with $C^{i} \in \mathcal{B}$ for all $i$ together with an isomorphism of cochain complexes

$$
\alpha_{C}: T^{2 p-2}\left(C^{*}\right) \longrightarrow C^{*}[2 p-2]=C^{*+2 p-2} .
$$

The morphisms are those morphisms of cochain complexes $f: C^{*} \rightarrow D^{*}$ that are compatible with the periodicity isomorphisms, that is, the following diagram commutes:

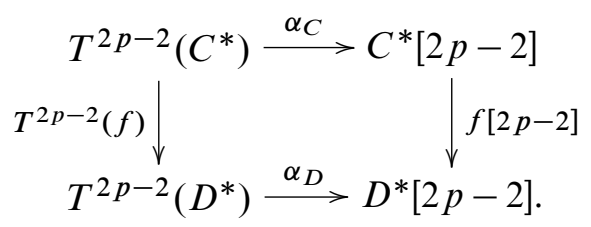

Such a cochain complex $C^{*}$ is called injective if each $C^{i}$ is injective in $\mathcal{B}$. A morphism in $\mathcal{C}^{2 p-2}(\mathcal{B})$ is called a quasi-isomorphism if it induces an isomorphism in cohomology. $C^{*}$ is called strictly injective if it is injective, and, for each acyclic complex $D^{*}$, the cochain complex $\operatorname{Hom}_{\mathcal{C}^{2 p-2}(\mathcal{B})}^{*}\left(D^{*}, C^{*}\right)$ is again acyclic.

Proposition 6.3 (Franke [7, 1.3.3, Proposition 3]) There is a model structure on $\mathcal{C}^{2 p-2}(\mathcal{B})$ such that

- weak equivalences are the quasi-isomorphisms,

- cofibrations are the monomorphisms,

- fibrations are the componentwise split epimorphisms with strictly injective kernel.

Notation $\mathcal{D}^{2 p-2}(\mathcal{B})$ denotes the derived category of $\mathcal{C}^{2 p-2}(\mathcal{B})$, that is, the homotopy category of this model category with respect to the above model structure.

Franke's functor $\mathcal{R}: \mathcal{D}^{2 p-2}(\mathcal{B}) \longrightarrow \operatorname{Ho}\left(L_{1} \mathcal{S}\right)$ now reconstructs a spectrum from the algebraic data given by $C^{*}$ for each twisted cochain complex $C^{*}$ over $\mathcal{B}$. The idea is to first associate a spectrum to each of the boundaries of $C^{*}$ and the quotients of $C^{*}$ by the boundaries. These spectra $X_{\beta_{i}}$ and $X_{\gamma_{i}}(1 \leq i \leq 2 p-2)$ are put into a diagram

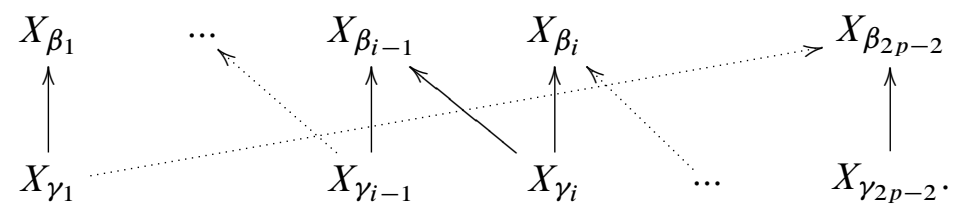

In the next step, the $X_{\beta_{i}}$ 's and $X_{\gamma_{i}}$ 's are pasted together by the homotopy colimit of this diagram. So all in all, the result is a spectrum $X=\mathcal{R}\left(C^{*}\right) \in \operatorname{Ho}\left(L_{1} \mathcal{S}\right)$ assigned to a twisted cochain complex $C^{*} \in \mathcal{D}^{2 p-2}(\mathcal{B})$. 
The condition that $p$ is odd is a special case of the condition that the splitting index of the abelian category $\mathcal{A}$ (into $2 p-2$ shifted copies of $\mathcal{B}$ ) is bigger than the injective dimension of $\mathcal{A}$, which is 2 . This ensures sparseness in certain Adams spectral sequences which the proof of Franke's theorem relies on. For details, see Franke [7, Section 2] and Roitzheim [16, Section 1-3].

Next, we note that

Proposition 6.4 The categories $\mathcal{D}^{2 p-2}(\mathcal{B})$ and $\operatorname{Ho}\left(L_{1} \mathcal{S}\right)$ are not Quillen equivalent. In particular, $\mathbb{R}$ is not derived from a Quillen equivalence.

Proof The proof of this relies on the mapping spaces with respect to $\mathcal{D}^{2 p-2}(\mathcal{B})$ and $\operatorname{Ho}\left(L_{1} \mathcal{S}\right)$. The categories $\mathcal{D}^{1}(\mathcal{A})$ and $\mathcal{D}^{2 p-2}(\mathcal{B})$ are not simplicial, but the technique of framings of Hovey [11, Chapter 5] allows the definition of a reasonable mapping space functor

$$
\operatorname{map}_{\mathcal{C}}(-,-): \operatorname{Ho}(\mathcal{C}) \times \operatorname{Ho}\left(\mathcal{C}^{o p}\right) \longrightarrow \operatorname{Ho}\left(\operatorname{sSet}_{*}\right)
$$

for any pointed model category $\mathcal{C}$.

A Quillen equivalence $F: \mathcal{C} \rightleftarrows \mathcal{D}: G$ would induce an isomorphism of mapping spaces

$$
\operatorname{map}_{\mathcal{C}}(X, Y) \stackrel{\sim}{\longrightarrow} \operatorname{map}_{\mathcal{D}}(L F(X), L F(Y))
$$

for $X, Y$ in $\mathcal{C}$.

Back to our special case: The category $\mathcal{C}^{2 p-2}(\mathcal{B})$ is abelian, so for all $C_{1}, C_{2} \in$ $\mathcal{C}^{2 p-2}(\mathcal{B})$, the $n$-simplices of $\operatorname{map}_{\mathcal{C}^{2 p-2}(\mathcal{B})}\left(C_{1}, C_{2}\right)$ form an abelian group, and the simplicial structure maps are group homomorphisms, so $\operatorname{map}_{\mathcal{C}^{2 p-2}(\mathcal{B})}\left(C_{1}, C_{2}\right)$ is not just a simplicial set but a simplicial abelian group. From Goerss and Jardine [8, Proposition III.2.20], it follows that $\operatorname{map}_{\mathcal{C}^{2 p-2}(\mathcal{B})}\left(C_{1}, C_{2}\right)$ is a product of EilenbergMac Lane spaces. However, there are spectra for which the mapping spaces over $L_{1} \mathcal{S}$ are not products of Eilenberg-Mac Lane spaces, for example $\operatorname{map}_{L_{1} \mathcal{S}}\left(S^{0}, S^{0}\right) \cong$ $Q L_{1} S^{0}=\operatorname{colim}_{n} \Omega^{n} L_{1} S^{n}$. Thus, $\mathcal{C}^{2 p-2}(\mathcal{B})$ and $L_{1} \mathcal{S}$ cannot be Quillen equivalent and $\mathcal{C}^{2 p-2}(\mathcal{B})$ provides an exotic model for $L_{1} \mathcal{S}$.

\subsection{A criterion for exotic models}

In this subsection, let $L_{1} \mathcal{S}$ denote the model category of spectra with the $K_{(p)}$-local model structure for $p>2$. How can we check in general whether a stable model category $\mathcal{C}$ provides an exotic model for $L_{1} \mathcal{S}$ or not?

In fact, we are going to prove that the answer to this question relies on the behaviour of just one element in the stable homotopy groups of the sphere: $\mathcal{C}$ is an exotic model for $\operatorname{Ho}\left(L_{1} \mathcal{S}\right)$ if and only if $X \wedge \alpha_{1}=0$ for $\alpha_{1} \in \pi_{2 p-3} S^{0}$ and $X \wedge-: \mathcal{S} \longrightarrow \mathcal{C}$ as before. 
The action of $\pi_{*} S^{0}$ on morphism sets We look at the action of $\pi_{*} S^{0}$ on the morphism sets of a stable homotopy category $\mathcal{C}$ in order to single out possible exotic models for $\operatorname{Ho}\left(L_{1} \mathcal{S}\right)$. In addition to the mapping space functor

$$
\operatorname{map}_{\mathcal{C}}(-,-): \operatorname{Ho}\left(\mathcal{C}^{o p}\right) \times \operatorname{Ho}(\mathcal{C}) \longrightarrow \operatorname{Ho}\left(\mathrm{sSet}_{*}\right)
$$

introduced in the proof of Proposition 6.4, one can use framings again to define a functor

$$
-\otimes-: \operatorname{Ho}(\mathcal{C}) \times \operatorname{Ho}(\text { set } *) \longrightarrow \operatorname{Ho}(\mathcal{C})
$$

such that

$$
A \otimes-: \operatorname{Ho}\left(\operatorname{sSet}_{*}\right) \rightleftarrows \mathrm{Ho}(\mathcal{C}): \operatorname{map}_{\mathcal{C}}(A,-)
$$

is an adjoint functor pair for $A \in \mathcal{C}$ (see Schwede and Shipley [18, Construction 2.4]). For $Y, Z \in \mathcal{C}$, we now use this functor to define a left action

$$
\pi_{n} S^{0} \otimes[Y, Z]_{k}^{\mathcal{C}} \stackrel{\mu}{\longrightarrow}[Y, Z]_{n+k}^{\mathcal{C}} .
$$

Let $f \in[Y, Z]_{k}^{\mathcal{C}}=\operatorname{Hom}_{\mathrm{Ho}(\mathcal{C})}^{0}\left(\Sigma^{k} Y, Z\right)=\operatorname{Hom}_{\mathrm{Ho}(\mathcal{C})}^{0}\left(Y \otimes \mathbb{S}^{k}, Z\right)$. (Note that the suspension functor $\Sigma$ defined in Section 2 is isomorphic to the functor $-\otimes \mathbb{S}^{1}$, with $\mathbb{S}^{1}$ denoting the simplicial 1-sphere.) For $\alpha \in \pi_{n} S^{0}$ we choose a representative $a: \mathbb{S}^{n+l} \longrightarrow \mathbb{S}^{l}$ in Ho(sSet $\left.*\right)$. The element $f \otimes a$ now lies in $\left[Y \otimes \mathbb{S}^{k} \otimes \mathbb{S}^{n+l}, Z \otimes \mathbb{S}^{l}\right]_{0}^{C}$ which is isomorphic to $[Y, Z]_{n+k}^{\mathcal{C}}$ since $\mathcal{C}$ is stable.

Definition 6.5 We now define $\mu(\alpha, f):=\alpha \cdot f$ to be the unique element in $[Y, Z]_{n+k}^{\mathcal{C}}$ such that $(\alpha \cdot f) \otimes \mathrm{id}_{\mathbb{S}^{l}}=f \otimes a$ in $\left[Y \otimes \mathbb{S}^{n+k+l}, Z \otimes \mathbb{S}^{l}\right]_{0}^{\mathcal{C}}$. (For details, see [18, Construction 2.4].)

Definition 6.6 Let $\mathcal{C}$ and $\mathcal{D}$ be stable model categories. A functor

$$
\Lambda: \operatorname{Ho}(\mathcal{C}) \longrightarrow \operatorname{Ho}(\mathcal{D})
$$

is called $\pi_{*} S^{0}$-exact [18, Definition 2.2] if $\Lambda$ is exact and $\pi_{*} S^{0}$-linear, that is, compatible with the $\pi_{*} S^{0}$-action.

There is an important example of a $\pi_{*} S^{0}$-linear functor: If $F: \mathcal{C} \longrightarrow \mathcal{D}$ is a left Quillen functor, then its left derived functor $L F: \operatorname{Ho}(\mathcal{C}) \longrightarrow \operatorname{Ho}(\mathcal{D})$ is $\pi_{*} S^{0}$-exact [18, Lemma 6.1]. Later in this section, we are going to apply this to the left Quillen functor $X \wedge-: \mathcal{S} \longrightarrow \mathcal{C}$ from the Universal Property of Spectra. 
Universal Property of $K_{(p)}$-local spectra Let us return to the proof of the Universal Property for $K_{(p)}$-local spectra and its first step, the question whether the spectra $\operatorname{Hom}(X, Y)$ are $K_{(p)}$-local for $Y \in \mathcal{C}$. This is again equivalent to the mod- $p$ homotopy groups of $\operatorname{Hom}(X, Y)$ being $v_{1}$-periodic (see Bousfield [2, Section 4]).

Let $M=M(\mathbb{Z} / p)$ denote the mod- $p$ Moore spectrum. For odd primes, the $v_{1}-$ self map of $M$ of smallest existing degree is not $v_{1}^{p^{2}}$ as in the case $p=2$, but

$$
v_{1}: \Sigma^{2 p-2} M \longrightarrow M
$$

itself. So a spectrum $\operatorname{Hom}(X, Y)$ is $K_{(p)}$-local if and only if the precomposition morphism

$$
\left(v_{1}\right)^{*}:[M, \operatorname{Hom}(X, Y)]_{n}^{\mathcal{S}} \longrightarrow[M, \operatorname{Hom}(X, Y)]_{n+2 p-2}^{\mathcal{S}}
$$

is an isomorphism for all $n$. By adjunction, this is equivalent to

$$
\left(X \wedge v_{1}\right)^{*}:[X \wedge M, Y]_{n}^{\mathcal{C}} \longrightarrow[X \wedge M, Y]_{n+2 p-2}^{\mathcal{C}}
$$

being an isomorphism for all $n$. The morphism $X \wedge v_{1}$ lies in

$$
[X \wedge M, X \wedge M]_{2 p-2}^{\mathcal{C}} \cong\left[\Phi\left(L_{1} M\right), \Phi\left(L_{1} M\right)\right]_{2 p-2}^{\mathcal{C}} \cong[M, M]_{2 p-2}^{L_{1} \mathcal{S}}=\mathbb{Z} / p\left\{v_{1}\right\} .
$$

So $\left(X \wedge v_{1}\right)^{*}$ is either an isomorphism or the zero map.

The element $\alpha_{1} \in \pi_{2 p-3} L_{1} S^{0}=\mathbb{Z} / p\left\{\alpha_{1}\right\}$ factors as

$$
\alpha_{1}=\text { pinch } \circ v_{1} \circ \text { incl, }
$$

which can be computed by similar methods to those in Section 5. It follows that $X \wedge v_{1}=0$ if and only if $X \wedge \alpha_{1}=0$. Let us investigate the two cases $X \wedge \alpha_{1}=0$ and $X \wedge \alpha_{1} \neq 0$ separately.

Case 1: $X \wedge \alpha_{1}=0$ We are going to prove that in this case, $\mathcal{C}$ and $L_{1} \mathcal{S}$ cannot be Quillen equivalent by using the $\pi_{*} S^{0}$-action defined earlier in this section.

The functor $X \wedge-: \mathcal{S} \longrightarrow \mathcal{C}$ from the Universal Property of Spectra is a left Quillen functor, and therefore its left derived $X \wedge-$ is $\pi_{*} S^{0}$-linear. (Again, we omit the $L$ for left derived from our notation.) So the diagram

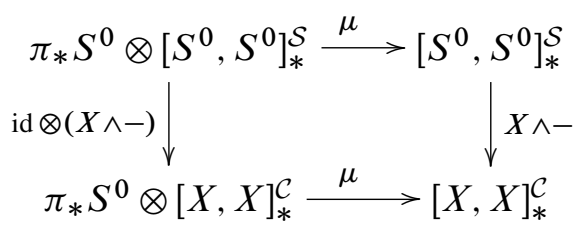


commutes. Consequently, for $\alpha \in \pi_{*} S^{0}$ we have

$$
\begin{aligned}
X \wedge \alpha & =X \wedge\left(\operatorname{Id}_{S^{0}} \cdot \alpha\right) \\
& =(X \wedge-) \circ \mu\left(\alpha, \operatorname{Id}_{S^{0}}\right) \\
& =\mu \circ(\mathrm{id} \otimes(X \wedge-))\left(\alpha, \operatorname{Id}_{S^{0}}\right) \\
& =\mu\left(\alpha, X \wedge \operatorname{Id}_{S^{0}}\right) \\
& =\mu\left(\alpha, \operatorname{Id}_{X}\right) \\
& =\alpha \cdot \operatorname{Id}_{X} .
\end{aligned}
$$

Let us assume that there is a functor

$$
F: L_{1} \mathcal{S} \longrightarrow \mathcal{C}
$$

that is part of a Quillen equivalence. (Without loss of generality, let $F$ be a left Quillen functor.) So then its derived functor $L F$ would be a $\pi_{*} S^{0}$-exact equivalence, in particular $\left[S^{0}, S^{0}\right]_{*}^{L_{1} \mathcal{S}}$ and $[X, X]_{*}^{\mathcal{C}}$ would be isomorphic as $\pi_{*} S^{0}$-modules. However, this cannot be the case as

$$
\alpha_{1} \cdot \operatorname{Id}_{X}=X \wedge \alpha_{1}=0 \quad \text { and } \quad \alpha_{1} \cdot \operatorname{Id}_{S^{0}}=\alpha_{1} \neq 0 .
$$

So we have shown

Proposition 6.7 If $X \wedge \alpha_{1}=0$, then $L_{1} \mathcal{S}$ and $\mathcal{C}$ are not Quillen equivalent.

Next, we will see that the condition $X \wedge \alpha_{1} \neq 0$ is both necessary and sufficient for the existence of a Quillen equivalence between $L_{1} \mathcal{S}$ and $\mathcal{C}$.

Case 2: $X \wedge \alpha_{1} \neq 0 \quad$ We have seen at the beginning of this subsection that $X \wedge \alpha_{1} \neq 0$ implies that the $\bmod -p$ homotopy groups of a spectrum $\operatorname{Hom}(X, Y)$ are $v_{1}$-periodic. Therefore, all $\operatorname{Hom}(X, Y)$ are $K_{(p)}$-local for $Y \in \mathcal{C}$. With the methods of Proposition 3.2 it now follows that

$$
X \wedge-: L_{1} \mathcal{S} \rightleftarrows \mathcal{C}: \operatorname{Hom}(X,-)
$$

is a Quillen functor pair for $X=\Phi\left(L_{1} S^{0}\right)$.

Analogously to Section 4, we now show that $\operatorname{Hom}(X, X)$ is equivalent to the $K_{(p)}-$ local sphere by showing that the map

$$
\lambda: L_{1} S^{0} \longrightarrow \operatorname{Hom}(X, X)
$$

is a $\pi_{*}$-isomorphism. Again, this is the case if and only if

$$
\Psi:\left[S^{0}, S^{0}\right]_{n}^{L_{1} \mathcal{S}} \stackrel{X \wedge^{L}-}{\longrightarrow}[X, X]_{n}^{\mathcal{C}} \stackrel{\Phi^{-1}}{\longrightarrow}\left[S^{0}, S^{0}\right]_{n}^{L_{1} \mathcal{S}}
$$


is an isomorphism for all $n \in \mathbb{Z}$. For $n=-1, \ldots, 2 p-1$ this follows easily as the only nontrivial homotopy groups of $L_{1} S^{0}$ in this range are $\pi_{0} L_{1} S^{0}=\mathbb{Z}_{(p)}\{l\}$ and $\pi_{2 p-3} L_{1} S^{0}=\mathbb{Z} / p\left\{\alpha_{1}\right\}$ (see Ravenel [14, 8.10.(b)]). By our assumption that $X \wedge \alpha_{1} \neq 0$ we can conclude that $\Psi\left(\alpha_{1}\right)$ is a nonzero multiple of $\alpha_{1}$. With the 5-lemma it follows that

$$
\Psi:\left[M, S^{0}\right]_{n}^{L_{1} \mathcal{S}} \longrightarrow\left[M, S^{0}\right]_{n}^{L_{1} \mathcal{S}}
$$

is an isomorphism for $n=0, \ldots, 2 p-2$.

We now use that $v_{1}: \Sigma^{2 p-2} M \longrightarrow M$ is an isomorphism in $\operatorname{Ho}\left(L_{1} \mathcal{S}\right)$, so by proceeding with exactly the same method as in Proposition 4.1 we conclude that $\lambda: L_{1} S^{0} \longrightarrow$ $\operatorname{Hom}(X, X)$ induces a $\pi_{*}$-isomorphism between $L_{1} S^{0}$ and $\operatorname{Hom}(X, X)$, and so, analogously to Theorem 4.2 ,

$$
X \wedge-: L_{1} \mathcal{S} \rightleftarrows \mathcal{C}: \operatorname{Hom}(X,-)
$$

is a Quillen equivalence for odd primes.

We summarize this subsection in the following theorem:

Theorem 6.8 Let $\Phi: \operatorname{Ho}\left(L_{1} \mathcal{S}\right) \longrightarrow \operatorname{Ho}(\mathcal{C})$ be an equivalence of triangulated categories, where $L_{1} \mathcal{S}$ denotes the category of spectra with the $K_{(p)}$-local model structure for $p$ odd. Then $L_{1} \mathcal{S}$ and $\mathcal{C}$ are Quillen equivalent if and only if

$$
X \wedge \alpha_{1} \neq 0, \quad \text { for } \quad X=\Phi\left(L_{1} S^{0}\right), \quad \alpha_{1} \in \pi_{2 p-3} S^{0}=\mathbb{Z} / p\left\{\alpha_{1}\right\} .
$$

However, while this Theorem tells us if a model $\mathcal{C}$ for $L_{1} \mathcal{S}$ is exotic or not, it does not answer the question of whether two exotic models are Quillen equivalent.

In particular, let us call a model algebraic if it is a model that is also an abelian category, such as Franke's example $\mathcal{C}=\mathcal{C}^{2 p-2}(\mathcal{B})$. An algebraic model is necessarily exotic by the mapping space argument given in the proof of Proposition 6.4. It would be interesting to find out if two algebraic models are automatically Quillen equivalent or not.

\section{Appendix A Proof of Lemma 3.4}

Lemma 3.4 For $\eta, v, \sigma, y_{0}, y_{1}$, and $\mu$ in $\pi_{*} L_{1} S^{0}$ as before, we have

$$
\begin{array}{rlrl}
X \wedge \eta & =\Phi(\eta) \text { or } \Phi(\eta)+\Phi\left(y_{1}\right) & X \wedge \mu & =\Phi(\mu) \text { or } \Phi(\mu)+\Phi\left(\eta^{2} \sigma\right) \\
X \wedge v & =u \Phi(v) \text { for some odd } u \in \mathbb{Z} & X \wedge \sigma & =\bar{u} \Phi(\sigma) \text { for some odd } \bar{u} \in \mathbb{Z} \\
X \wedge y_{0} & =\Phi\left(y_{0}\right) & X \wedge y_{1} & =\Phi\left(y_{1}\right) .
\end{array}
$$


Proof $X \wedge \eta$ : On the mod-2 Moore spectrum $M, 2 \operatorname{Id}_{M}$ factors as

$$
M \stackrel{\text { pinch }}{\longrightarrow} S^{1} \stackrel{\eta}{\longrightarrow} S^{0} \stackrel{\text { incl }}{\longrightarrow} M,
$$

and this composition is nonzero. Here, pinch denotes the map that "pinches" off the bottom cell of $M$, and incl denotes the inclusion of the zero-sphere into the bottom cell of $M$. Consequently, $2 \operatorname{Id}_{L_{1}} M$ factors as

$$
L_{1} M \stackrel{\text { pinch }}{\longrightarrow} L_{1} S^{1} \stackrel{\eta}{\longrightarrow} L_{1} S^{0} \stackrel{\text { incl }}{\longrightarrow} L_{1} M .
$$

Recall that $\eta$ survives $K_{(2)}$-localisation. We now consider the exact triangle

$$
S^{0} \stackrel{2}{\longrightarrow} S^{0} \stackrel{\text { incl }}{\longrightarrow} M \stackrel{\text { pinch }}{\longrightarrow} S^{1}
$$

in $\operatorname{Ho}(\mathcal{S})$. The functor $X \wedge-: \operatorname{Ho}(\mathcal{S}) \longrightarrow \operatorname{Ho}(\mathcal{C})$ is exact on the homotopy level, $\Phi$ is exact and $X \wedge S^{0} \cong \Phi\left(L_{1} S^{0}\right)$, so we can choose an isomorphism

$$
X \wedge M \cong \Phi\left(L_{1} M\right)
$$

such that $X \wedge$ pinch corresponds to $\Phi($ pinch) and $X \wedge$ incl corresponds to $\Phi($ incl) .

The functor $X \wedge-$ is additive on the homotopy level, so

$$
X \wedge 2 \operatorname{Id}_{L_{1} M}=2 \operatorname{Id}_{X \wedge M} \neq 0,
$$

since

$$
[X \wedge M, X \wedge M]_{0}^{\mathcal{C}} \cong\left[\Phi\left(L_{1} M\right), \Phi\left(L_{1} M\right)\right]_{0}^{\mathcal{C}} \cong[M, M]_{0}^{L_{1} \mathcal{S}} \cong \mathbb{Z} / 4\left\{\operatorname{Id}_{L_{1} M}\right\} .
$$

Furthermore, $2 \operatorname{Id}_{X \wedge M}$ factors as

$$
X \wedge M \stackrel{X \wedge \text { pinch }}{\longrightarrow} X \wedge S^{1} \stackrel{X \wedge \eta}{\longrightarrow} X \wedge S^{0} \stackrel{X \wedge \text { incl }}{\longrightarrow} X \wedge M .
$$

Consequently,

$$
X \wedge \eta \in[X, X]_{1}^{\mathcal{C}} \cong \mathbb{Z} / 2\left\{\Phi(\eta), \Phi\left(y_{1}\right)\right\}
$$

cannot be zero.

Also, $X \wedge \eta$ cannot be $\Phi\left(y_{1}\right)$ either: the composition

$$
L_{1} M \stackrel{\text { pinch }}{\longrightarrow} L_{1} S^{1} \stackrel{y_{1}}{\longrightarrow} L_{1} S^{0} \stackrel{\text { incl }}{\longrightarrow} L_{1} M
$$

is zero by equation (2) in Section 5. So if $X \wedge \eta=\Phi\left(y_{1}\right)$, then 


$$
\begin{aligned}
2 \operatorname{Id}_{X \wedge M} & =(X \wedge \text { incl }) \circ(X \wedge \eta) \circ(X \wedge \text { pinch }) \\
& =\Phi(\text { incl }) \circ \Phi\left(y_{1}\right) \circ \Phi(\text { pinch }) \\
& =\Phi\left(\text { incl } \circ y_{1} \circ \text { pinch }\right)=\Phi(0)=0,
\end{aligned}
$$

which is a contradiction to (6). It follows that either

$$
X \wedge \eta=\Phi(\eta) \quad \text { or } \quad X \wedge \eta=\Phi(\eta)+\Phi\left(y_{1}\right) .
$$

$X \wedge v$ : Whether $X \wedge \eta=\Phi(\eta)$ or $X \wedge \eta=\Phi(\eta)+\Phi\left(y_{1}\right)$, we have

$$
X \wedge \eta^{3}=(X \wedge \eta)^{3}=\Phi(\eta)^{3} .
$$

as $\eta y_{1}$ and $y_{1}^{2}$ are both zero in $\pi_{*} L_{1} S^{0}$. Since in $\pi_{3} L_{1} S^{0}$ there is the relation $\eta^{3}=4 v$, we have

$$
4(X \wedge v)=X \wedge \eta^{3}=4 \Phi(v) .
$$

As $4 \Phi(v) \neq 0$ in $[X, X]_{3}^{\mathcal{C}} \cong \mathbb{Z} / 8\{\Phi(v)\}, X \wedge v$ has order eight in this group and is therefore a generator. Consequently

$$
X \wedge v=u \Phi(v), \quad \text { for some odd integer } u \in \mathbb{Z} .
$$

$X \wedge \sigma:$ For $X \wedge \sigma$ we look at the Toda bracket relation

$$
8 \sigma=\langle\nu, 8, v\rangle
$$

From this we obtain

$$
X \wedge 8 \sigma \in\langle X \wedge v, X \wedge 8, X \wedge v\rangle .
$$

The indeterminacy of this Toda bracket is zero, thus, equality holds. By the computations above, we get

$$
8(X \wedge \sigma)=X \wedge 8 \sigma=\langle u \Phi(v), \Phi(8), u \Phi(v)\rangle=u^{2} \Phi(8 \sigma)
$$

which is nonzero in $[X, X]_{7}^{\mathcal{C}} \cong \mathbb{Z} / 16\{\Phi(\sigma)\}$. We conclude that $X \wedge \sigma$ has order 16 in this group, so

$$
X \wedge \sigma=\bar{u} \Phi(\sigma), \quad \text { for some odd integer } \bar{u} \in \mathbb{Z} .
$$

$X \wedge \mu$ : Next, we use that $\mu \in\langle 2,8 \sigma, \eta\rangle$ with indeterminacy $\eta^{2} \sigma$. It follows that

$$
X \wedge \mu \in\langle X \wedge 2, X \wedge 8 \sigma, X \wedge \eta\rangle .
$$

Using our previous computations, this bracket either equals $\langle 2, \Phi(8 \sigma), \Phi(\eta)\rangle$ or $\left\langle 2, \Phi(8 \sigma), \Phi(\eta)+\Phi\left(y_{1}\right)\right\rangle$. 
In the first case, $\langle 2, \Phi(8 \sigma), \Phi(\eta)\rangle=\Phi(\langle 2,8 \sigma, \eta\rangle)=\left\{\Phi(\mu), \Phi(\mu)+\Phi\left(\eta^{2} \sigma\right)\right\}$ which is what we want.

For the second case, we compute

$$
\left\langle 2,8 \sigma, \eta+y_{1}\right\rangle \supseteq\langle 2,8 \sigma, \eta\rangle+\left\langle 2,8 \sigma, y_{1}\right\rangle .
$$

The bracket on the left side has indeterminacy $\eta^{2} \sigma$, which is the same as the intederminacy of the first bracket on the right side. The last bracket has indeterminacy zero and contains the set $\langle 2,8 \sigma, \eta\rangle y_{0}=\left\{\mu y_{0}, \mu y_{0}+\eta^{2} \sigma y_{0}\right\}=\left\{\eta^{2} \sigma\right\}$ (by the relations given in (1), so it equals $\left\{\eta^{2} \sigma\right\}$. Thus, equality holds in (11), and we can also conclude in this case that

$$
X \wedge \mu \in\left\{\Phi(\mu), \Phi(\mu)+\Phi\left(\eta^{2} \sigma\right)\right\}
$$

$X \wedge y_{0}$ : Next, we look at $X \wedge y_{0}$. Since $y_{0}$ is the only nonzero torsion element in $\pi_{0} L_{0} S^{0}=\mathbb{Z}_{(2)} \oplus \mathbb{Z} / 2$, the element $X \wedge y_{0}$ must be a torsion element as well, because the functor $X \wedge-$ is additive. Consequently, $X \wedge y_{0}$ either equals $\Phi\left(y_{0}\right)$ or zero.

We now make use of the multiplicative relation $\mu y_{0}=\eta^{2} \sigma$ (see Ravenel $[14,8.15$.(d)]). We have already seen that $X \wedge \eta^{2} \sigma=\bar{u} \Phi\left(\eta^{2} \sigma\right) \neq 0$, so $X \wedge y_{0}$ cannot be zero. Consequently,

$$
X \wedge y_{0}=\Phi\left(y_{0}\right) .
$$

$X \wedge y_{1}$ : Now determining $X \wedge y_{1}$ is easy: we have $y_{1}=\eta y_{0}$, so

$$
X \wedge y_{1}=(X \wedge \eta)\left(X \wedge y_{0}\right)=\Phi(\eta) \Phi\left(y_{0}\right) \quad \text { or } \quad=\Phi(\eta) \Phi\left(y_{0}\right)+\Phi\left(y_{0}^{2}\right)
$$

which in either case equals $\Phi\left(y_{1}\right)$ since $y_{0}^{2}=0$ by Ravenel [14, 8.15.(d)].

\section{References}

[1] A K Bousfield, The localization of spaces with respect to homology, Topology 14 (1975) 133-150 MR0380779

[2] A K Bousfield, The localization of spectra with respect to homology, Topology 18 (1979) 257-281 MR551009

[3] A K Bousfield, On the homotopy theory of $K$-local spectra at an odd prime, Amer. J. Math. 107 (1985) 895-932 MR796907

[4] A K Bousfield, E M Friedlander, Homotopy theory of $\Gamma$-spaces, spectra, and bisimplicial sets, from: "Geometric applications of homotopy theory (Proc. Conf., Evanston, Ill., 1977), II", Lecture Notes in Math. 658, Springer, Berlin (1978) 80-130 MR513569 
[5] F Clarke, M Crossley, $\mathbf{S}$ Whitehouse, The discrete module category for the ring of K-theory operations, Topology 46 (2007) 139-154 MR2313069

[6] D Dugger, Replacing model categories with simplicial ones, Trans. Amer. Math. Soc. 353 (2001) 5003-5027 MR1852091

[7] J Franke, Uniqueness theorems for certain triangulated categories possessing an adams spectral sequence, preprint (1996) Available at http://www.math.uiuc.edu/ K-theory/0139/

[8] P G Goerss, J F Jardine, Simplicial homotopy theory, Progress in Mathematics 174, Birkhäuser Verlag, Basel (1999) MR1711612

[9] PS Hirschhorn, Model categories and their localizations, Mathematical Surveys and Monographs 99, American Mathematical Society, Providence, RI (2003) MR1944041

[10] M J Hopkins, J H Smith, Nilpotence and stable homotopy theory II, Ann. of Math. (2) 148 (1998) 1-49 MR1652975

[11] M Hovey, Model categories, Mathematical Surveys and Monographs 63, American Mathematical Society, Providence, RI (1999) MR1650134

[12] B Keller, Deriving DG categories, Ann. Sci. École Norm. Sup. (4) 27 (1994) 63-102 MR1258406

[13] D G Quillen, Homotopical algebra, Lecture Notes in Mathematics 43, Springer, Berlin (1967) MR0223432

[14] D C Ravenel, Localization with respect to certain periodic homology theories, Amer. J. Math. 106 (1984) 351-414 MR737778

[15] D C Ravenel, Complex cobordism and stable homotopy groups of spheres, Pure and Applied Mathematics 121, Academic Press, Orlando, FL (1986) MR860042

[16] C Roitzheim, On the algebraic classification of $K$-local spectra arXiv: 0708.3036v1

[17] S Schwede, The stable homotopy category is rigid, preprint (2005) Available at http://www . math.uni-bonn.de/people/schwede/rigid.pdf

[18] S Schwede, B Shipley, A uniqueness theorem for stable homotopy theory, Math. Z. 239 (2002) 803-828 MR1902062

[19] H Toda, Composition methods in homotopy groups of spheres, Annals of Mathematics Studies 49, Princeton University Press, Princeton, N.J. (1962) MR0143217

Department of Pure Mathematics, University of Sheffield

Hicks Building, Hounsfield Road, Sheffield, S3 7RH, UK

C.Roitzheim@sheffield.ac.uk

http://www.roitzheim.staff.shef.ac.uk/

Proposed: Paul Goerss

Received: 5 April 2007

Seconded: Haynes Miller, Bill Dwyer

Revised: 23 August 2007

Geometry 85 Topology, Volume 11 (2007) 